\title{
From stellar coronae to gyrochronology: A theoretical and observational exploration
}

\author{
J. Ahuir, A. S. Brun, and A. Strugarek

\begin{abstract}
Département d'Astrophysique-AIM, CEA/DRF/IRFU, CNRS/INSU, Université Paris-Saclay, Université Paris-Diderot, Université de Paris, 91191 Gif-sur-Yvette, France

e-mail: jeremy.ahuir@cea.fr
\end{abstract}

Received 22 October 2019 / Accepted 3 February 2020

\begin{abstract}
Context. Stellar spin down is the result of a complex process involving rotation, dynamo, wind, and magnetism. Multiwavelength surveys of solar-like stars have revealed the likely existence of relationships between their rotation, X-ray luminosity, mass losses, and magnetism. They impose strong constraints on the corona and wind of cool stars.

Aims. We aim to provide power-law prescriptions of the mass loss of stars, of their magnetic field, and of their base coronal density and temperature that are compatible with their observationally-constrained spin down.

Methods. We link the magnetic field and the mass-loss rate from a wind torque formulation, which is in agreement with the distribution of stellar rotation periods in open clusters and the Skumanich law. Given a wind model and an expression of the X-ray luminosity from radiative losses, we constrained the coronal properties by assuming different physical scenarios linking closed loops to coronal holes.

Results. We find that the magnetic field and the mass loss are involved in a one-to-one correspondence that is constrained from spin down considerations. We show that a magnetic field, depending on both the Rossby number and the stellar mass, is required to keep a consistent spin down model. The estimates of the magnetic field and the mass-loss rate obtained from our formalism are consistent with statistical studies as well as individual observations and they give new leads to constrain the magnetic field-rotation relation. The set of scaling-laws we derived can be broadly applied to cool stars from the pre-main sequence to the end of the main sequence (MS), and they allow for stellar wind modeling that is consistent with all of the observational constraints available to date.
\end{abstract}

Key words. stars: rotation - stars: magnetic field - stars: mass-loss - stars: winds, outflows - stars: solar-type

\section{Introduction}

The rotation of stars is subject to complex evolution during their whole life. During the early stage of their lifetime, solar-type stars spin up as they contract during the pre-main sequence. Once the zero-age main sequence (ZAMS) is reached, their moment of inertia remains relatively constant (Armitage \& Clarke 1996) while they lose mass and angular momentum through the flow of a magnetized stellar wind (Schatzman 1962; Weber \& Davis 1967; Mestel 1968). It results in a slow-down of their rotation as they age, which approximately follows the empirical Skumanich's law: $\Omega_{\star} \propto t^{-0.5}$ (Skumanich 1972). This spin down also depends on the stellar mass (Weber \& Davis 1967; Matt et al. 2015). Indeed, during most of the pre-main sequence, lower mass stars tend to remain fast rotators for a longer time than the higher mass stars. Then, after hundreds of millions of years, the slowest rotators converge toward a sequence in which the rotation rate increases with mass. These phenomena make gyrochronology possible (Barnes 2003), thereby allowing for the estimation of stellar ages through measurements of rotation periods and masses.

Understanding the feedback loop between rotation, dynamo action, magnetism, and wind is a key issue in predicting the behavior of the solar-like stars as they evolve. It is also important to understand the evolution of star-planet systems and to follow potential planetary migrations (Zhang \& Penev 2014; Benbakoura et al. 2019). Our ability to track the stellar rotation evolution strongly relies on the wind braking modeling.
Most angular momentum evolution models rely on the Kawaler (1988) prescription when assessing the wind torque, which is expressed in this model as a power-law depending on the magnetic field, the mass-loss rate, the mass, and the radius of the star. Several modifications have since been added to this formulation (Krishnamurthi et al. 1997; Bouvier et al. 1997; Reiners \& Mohanty 2012). For instance, to account for fast rotators on the ZAMS, a saturation of the braking torque is required (Barnes \& Sofia 1996). Magnetohydrodynamical (MHD) simulations can also be used to assess of the angular momentum loss due to the wind. For instance, Matt et al. (2012), following Matt \& Pudritz (2008), simulated the flow of a stellar wind along the opened field lines of a dipolar configuration to estimate the torque by taking the influence of stellar rotation on the wind acceleration into account (Sakurai 1985). More recently, modified versions of this formulation have been presented to take the influence of the magnetic topology into account. Réville et al. (2015a) considered the magnetic flux through the open field lines to build a topology-independant wind torque, while Finley \& Matt $(2017,2018)$ relied on a broken powerlaw behavior to deal with combined geometries. Garraffo et al. (2016, 2018) accounted for magnetic topology by means of a modulating factor to the angular momentum loss estimated for a dipolar configuration. In general, in most of the prescriptions of spin-evolution torques, it is assumed that the wind carries away angular momentum at a rate proportional to $\Omega_{\star}^{3}$ during the main sequence in order to follow the Skumanich law. It is important to 
note that the Skumanich law is currently questioned for evolved stars. Some recent studies have shown a substantial decrease of the wind braking efficiency for evolved stars around the solar value of the Rossby number (van Saders et al. 2016) even if this alternative scenario seems to be in disagreement with solar twin studies (Lorenzo-Oliveira et al. 2019).

To estimate the angular momentum loss, we need to know the precise stellar magnetic field and the accurate properties of its wind (such as the mass loss induced). The latter is bound to physical parameters in the corona, such as the plasma temperature and density. Several observational trends constrain and correlate those different quantities: the mass loss (Wood et al. 2002, 2005; Jardine \& Collier 2019), the X-ray activity (Pizzolato et al. 2003; Wright et al. 2011; Reiners et al. 2014), the magnetic field (Vidotto et al. 2014; See et al. 2017), and the rotation rate of the star for different ages (Agüeros et al. 2011; McQuillan et al. 2014; Gallet \& Bouvier 2015). Coupling all those quantities is therefore necessary when designing a consistent model of stellar spin down.

A preliminary exploration was carried out by Blackman \& Owen (2016), who present a simplified model for the coupled time evolution of the relevant quantities on the basis of a pressure-driven isothermal wind (Parker 1958). In this framework, a dynamo-induced magnetic field dictates the behavior of the stellar wind and the X-ray luminosity through a coronal equilibrium. More recently, Skumanich (2019) has focused on the connections between the physical parameters regulating the stellar spin down by considering their rotational evolution. By assuming a direct correlation between the mass loss and the magnetic field, he studied the influence of the Skumanich law on all of the relevant quantities by means on a variety of observational trends. Such a study suggests that the magnetic field and the rotation of a cool star should scale linearly, while the mass loss should scale quadratically with stellar rotation.

More generally, stellar wind models require knowledge on the coronal temperature and density. To be consistent with the aforementioned correlations, one needs to constrain those two quantities from tracers of the coronal activity, such as the soft X-ray emission of the star. In this spirit, by relying on a 1D polytropic and magnetocentrifugal wind, Holzwarth \& Jardine (2007) provided scaling laws that are in accordance with the rotational evolution of the X-ray luminosity (Ivanova \& Taam 2003) and the empirical mass loss-X-ray flux correlation from Wood et al. (2005).

In building on those previous studies, the main goal of this paper is to infer from stellar spin down considerations some prescriptions of the magnetic field, the mass loss, the coronal temperature, and the coronal density as a function of fundamental stellar parameters (such as mass, radius, and rotation rate) in order to be consistent with all of the observational trends. Each of those constraints are successively introduced to eliminate the largest number of free parameters involved in the spin down process. In our attempt to extract the most important interdependencies between the various physical mechanisms involved in the stellar spin down theory, we had to make some simplifying assumptions; however, we were careful to retain all of the key mechanisms. Our scaling laws provide a novel and systematic way to connect all of these mechanisms together and they should be seen as a first approach to systematically characterize these complex relationships between the stellar parameters. In Sect. 2 we introduce the theoretical framework we used for the torque parametrization. In Sect. 3, observational constraints are leveraged to unveil the inter-dependency of the magnetic field and the mass-loss rate of cool stars. We further derived the associated prescriptions for the coronal temperature and the coronal density for a given wind model. In Sect. 4, we summarize our prescriptions and give a practical application in the case of $\epsilon$ Eridani. All of those results are then summarized, discussed, and put in perspective in Sect. 5.

\section{Stellar wind torques of solar-type stars: Theoretical approach}

\subsection{Fundamental stellar parameters and architecture of the model}

First of all, designing a consistent model for the stellar spin down requires taking an inventory of the coupling between the various physical mechanisms, and their associated control parameters, involved in the process. Stellar rotation and magnetism strengthen the supersonic flow of a stellar wind (Weber \& Davis 1967), which itself entails a mass loss of $\dot{M}$. The wind then carries away angular momentum, leading to a braking torque $\Gamma_{\text {wind }}$ that spins down the star. The logical sequence from fundamental stellar parameters to the wind braking torque and the role of the X-ray luminosity inside the architecture of the model is summarized in Fig. 1, where we show all of the interdependencies between the various physical mechanisms and their control parameters. Theoretical assumptions are represented in blue and are essentially related to the wind torque parametrization, dynamo scalings, as well as the choice of a wind model. Observational trends, which are in red, are considered to be constraints for the physical models (see Appendix F for an extensive view of those ingredients and their caveats).

Solar-type stars generate a magnetic field through a dynamo in their envelope (Brun et al. 2004, 2015) that is triggered by turbulent convective movements, which can be influenced by stellar rotation (Durney \& Latour 1978). This effect can be quantified by the Rossby number Ro, which has been shown to be a relevant quantity in characterizing the magnetic activity of the stars (Noyes et al. 1984). Direct spectropolarimetric studies also exhibit scaling laws linking the stellar magnetic field and this dimensionless number (Vidotto et al. 2014; See et al. 2017). However, multiple definitions of the Rossby number are available in the literature, which has forced the community to clarify this aspect (Landin et al. 2010; Brun et al. 2017; Amard et al. 2019). In this work, the Rossby number is normalized to the solar value. This way, different prescriptions of the Rossby number can be equally used as long as they only differ by a proportionality factor. This is generally the case of main-sequence stars, which we focus on here. However, to deal with individual stars, we used the stellar Rossby number for simplicity purposes, which is expressed as

Ro $=\frac{P_{\text {rot }}}{\tau_{\mathrm{c}}}$

where $P_{\text {rot }}$ is the stellar rotation period and $\tau_{\mathrm{c}}$ is the convective turnover time. The latter itself can be assessed at different locations or computed in a variety of ways, which are often linked to a given stellar evolution model (Landin et al. 2010; Cranmer \& Saar 2011; Sadeghi Ardestani et al. 2017) or a set of observations (Wright et al. 2011).

Coronal activity, which is measured with the X-ray emission of the star, has been correlated with the stellar magnetic field (Pevtsov et al. 2003; Vidotto et al. 2014) and the mass loss (Wood et al. 2002, 2005). Observations have also exhibited a relationship between the stellar rotation and the 


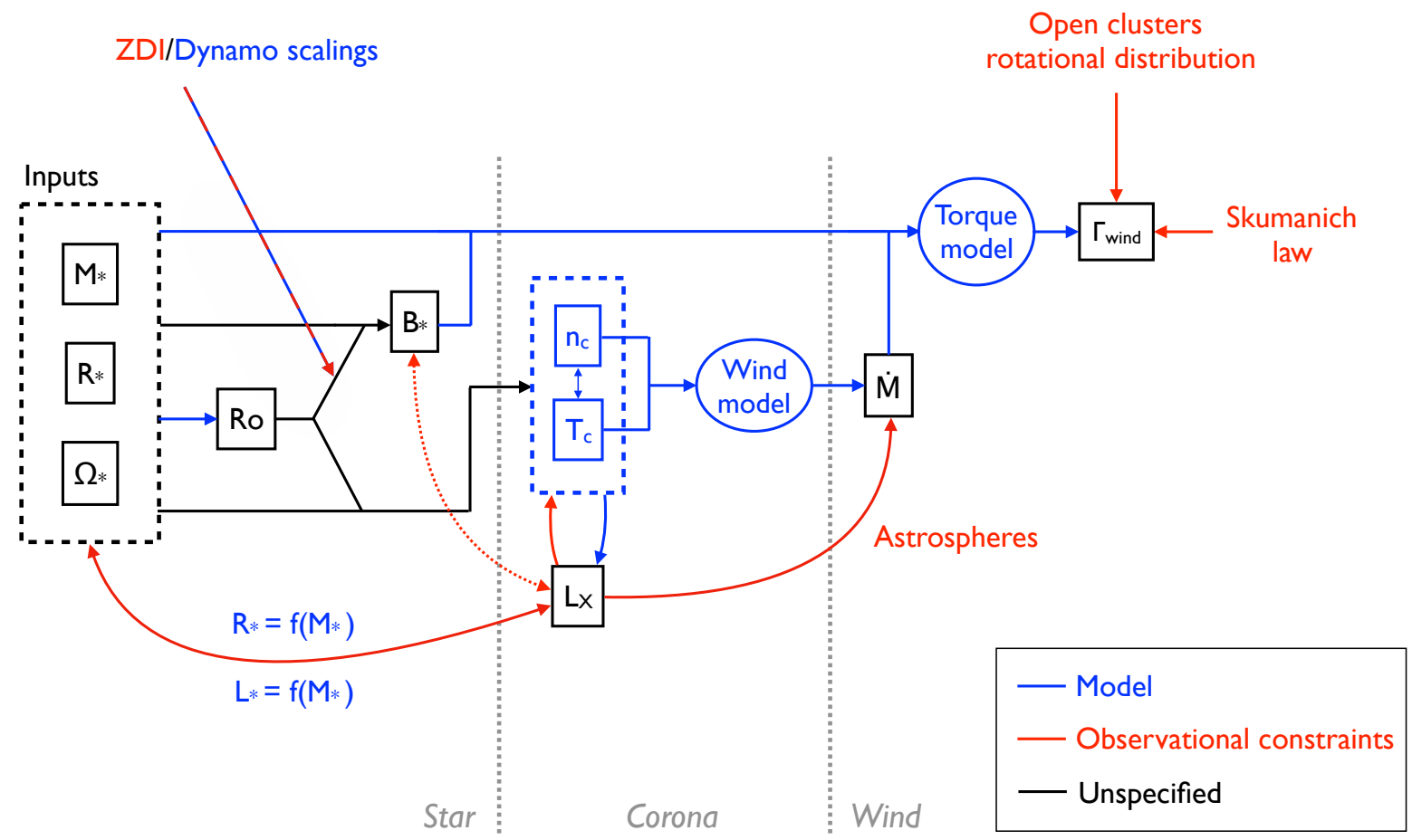

Fig. 1. Sketch of the multiple couplings between the inputs of the model (stellar mass, radius, and rotation rate), the magnetic field, the X-ray luminosity, the coronal temperature and density, the mass loss, and the wind braking torque. In blue: model assumptions. In red: observational constraints.

X-ray luminosity for main-sequence stars (Noyes et al. 1984; Pizzolato et al. 2003; Wright et al. 2011; Vidotto et al. 2014; Reiners et al. 2014), allowing us to link the coronal activity to fundamental stellar parameters. This gives us the opportunity to closely link those physical quantities together through stellar activity considerations.

Furthermore, the corona of the stars that we consider is thought to be heated up through magnetic processes (for a review, see Mathioudakis et al. 2013; Cranmer et al. 2017) to reach a typical coronal temperature $T_{\mathrm{c}}$ of around $10^{6} \mathrm{~K}$ (Suzuki \& Inutsuka 2006). Such a hot and diluted medium (with a density $n_{\mathrm{c}}$ ) expands and generates a transsonic wind (Parker 1958). Observationally, scaling laws have been established between the coronal temperature and the X-ray emission of the star (Preibisch 1997; Johnstone \& Güdel 2015; Wood et al. 2018). From a theoretical point of view, the latter can be deduced from coronal properties by estimating the radiative losses in the X-ray emitting area of the corona (Ivanova \& Taam 2003; Aschwanden 2004; Blackman \& Owen 2016). Therefore, it could be possible to provide a prescription for the coronal temperature and density in terms of the stellar mass, radius, and rotation rate.

\subsection{Torque parametrization}

Solar-like stars spin down due to the angular momentum extraction by the stellar wind (Schatzman 1962). Weber \& Davis (1967) showed with a 1D model in the stellar equatorial plane that the angular momentum loss $\Gamma_{\text {wind }}$ can be estimated at the Alfvén radius $r_{\mathrm{A}}$ as

$\Gamma_{\text {wind }}=\dot{M} r_{\mathrm{A}}^{2} \Omega_{\star}$

in which $\Omega_{\star}$ is the stellar rotation rate and $\dot{M}$ is the mass loss. The Alfvén radius can be expressed as a function of the stellar magnetic field, the mass loss, and other fundamental stellar parameters, such as the stellar mass, the stellar radius, and the stellar rotation rate (Kawaler 1988). For instance, Matt et al. (2012) present the following expression for this characteristic distance by assuming a dipolar magnetic field:

$\frac{\left\langle r_{\mathrm{A}}\right\rangle}{R_{\star}}=K_{1}\left[\frac{\Upsilon}{\sqrt{K_{2}^{2}+0.5 f^{2}}}\right]^{m}$,

where $M_{\star}$ is the stellar mass, $R_{\star}$ is the stellar radius, and $K_{1}$, $K_{2}$, and $m$ are constants, which were set using 2D MHD simulations. More precisely, $K_{1}$ is used to calibrate the solar wind torque, $K_{2}$ is the efficiency of the magnetocentrifugal acceleration (Sakurai 1985), and $m$ stands for a magnetic topology parameter (Réville et al. 2015a). For a dipolar field, we use the value proposed in Matt et al. (2012), that is, $m=0.2177$. We note that $f=\Omega_{\star} / \sqrt{G M_{\star} / R_{\star}^{3}}$ is the break-up ratio, which is obtained by dividing the stellar rotation rate at the equator of the star by the Keplerian angular velocity. The magnetization parameter $\Upsilon$ (Matt \& Pudritz 2008) is defined as

$\Upsilon=\frac{B_{\star}^{2} R_{\star}^{2}}{\dot{M} v_{\mathrm{esc}}}$

where $B_{\star}$ is the magnetic field strength at the stellar equator and $v_{\text {esc }}=\sqrt{2 G M_{\star} / R_{\star}}$ is the escape velocity. With this formulation, the wind braking torque becomes

$\begin{aligned} \Gamma_{\text {wind }}= & \dot{M} \Omega_{\star} R_{\star}^{2} K_{1}^{2}\left[\frac{\Upsilon}{\sqrt{K_{2}^{2}+0.5 f^{2}}}\right]^{2 m} \\ & \propto \dot{M}^{1-2 m} B_{\star}^{4 m} R_{\star}^{2+5 m} M_{\star}^{-m} \Omega_{\star}\left(K_{2}^{2}+0.5 f^{2}\right)^{-m} .\end{aligned}$ 
We rewrite this power-law expansion of the torque to adopt the following generic expression

$\Gamma_{\text {wind }} \propto \dot{M}^{1-2 m} B_{\star}^{4 m} R_{\star}^{2+5 m} M_{\star}^{-m} \Omega_{\star}\left[1+\frac{f^{2}}{K^{2}}\right]^{-m}$,

where $K=\sqrt{2} K_{2}$ is a constant.

\subsection{Stellar magnetic field prescription}

Computing the wind braking torque requires estimating the stellar magnetic field and the mass-loss rate. Here, we focus on the surface magnetic field strength at the stellar equator $B_{\star}$. For the sake of simplicity, we assume a power-law expression for the magnetic field as a function of the stellar mass, the stellar radius, and the Rossby number as

$B_{\star} \propto\left(\frac{\mathrm{Ro}}{\mathrm{Ro}_{\odot}}\right)^{-p_{B}}\left(\frac{R_{\star}}{R_{\odot}}\right)^{r_{B}}\left(\frac{M_{\star}}{M_{\odot}}\right)^{m_{B}}$,

where $p_{B}, m_{B}$, and $r_{B}$ are unspecified exponents for the time being.

Since we present a significant number of power-law prescriptions in the rest of this work, we have to fix a generic notation for the different exponents. More precisely, for each estimate of a physical quantity, we write a given exponent with a lowercase letter indicating the variable of the power law ( $p$ for the rotation period, $r$ for the stellar radius, and $m$ for the stellar mass). Each letter has a subscript in capital letters representing the quantity for which we give a prescription. For example, to express a quantity $A$ as a function of the stellar mass $M_{\star}$, we write

$A \propto M_{\star}^{m_{\mathrm{A}}}$.

\subsection{Mass loss prescription}

Because of the wind torque parametrization and the stellar magnetic field prescription, we consider a power-law expression of the stellar mass loss for the sake of consistency, as follows:

$\dot{M} \propto\left(\frac{\mathrm{Ro}}{\mathrm{Ro}_{\odot}}\right)^{-p_{\dot{M}}}\left(\frac{R_{\star}}{R_{\odot}}\right)^{r_{M}}\left(\frac{M_{\star}}{M_{\odot}}\right)^{m_{M}}$,

where $p_{\dot{M}}, m_{\dot{M}}$, and $r_{\dot{M}}$ are constrained later. Those exponents follow the nomenclature presented in Sect. 2.3. However, the mass loss can be obtained from coronal quantities through a wind model, for example, by assuming a radial polytropic pressure-driven outflow, with an index $\gamma$ (cf. Appendix A):

$\dot{M} \propto\left(\frac{M_{\star}}{M_{\odot}}\right)^{2}\left(\frac{n_{\mathrm{c}}}{n_{\odot}}\right)\left(\frac{T_{\mathrm{c}}}{T_{\odot}}\right)^{-\frac{3}{2}}\left[1-\frac{T_{\min , \odot}}{T_{\odot}} \frac{M_{\star}}{M_{\odot}} \frac{R_{\odot}}{R_{\star}} \frac{T_{\odot}}{T_{\mathrm{c}}}\right]^{\frac{5-3 \gamma}{2(\gamma-1)}}$

where $n_{\odot}, T_{\odot}$ are respectively the solar values of the density and temperature at the base of the corona. We note that $T_{\min , \odot}=$ $(1-1 / \gamma) G m_{\mathrm{p}} M_{\odot} / 2 k_{\mathrm{B}} R_{\odot} \approx 11(1-1 / \gamma) \mathrm{MK}$ is the minimal temperature needed at the base of the corona for the Sun to obtain a transsonic wind. For instance, for $\gamma=1.05$, which is the value commonly used in the literature (Matt et al. 2012; Réville et al. 2015a; Finley \& Matt 2017), we have $T_{\min , \odot} \approx 0.52 \mathrm{MK}$. Such an expression is used in Sect. 3.5.

\subsection{General formulation of the torque}

The parametrization of the torque with Eq. (6), together with the power-law expressions of the stellar magnetic field and the mass loss (Eqs. (7) and (9)) lead to the following formulation of the torque as a function of fundamental stellar parameters:

$$
\begin{aligned}
\Gamma_{\text {wind }} \propto & \mathrm{Ro}^{-4 m \cdot p_{B}-(1-2 m) p_{\dot{M}}} R_{\star}^{2+5 m+4 m \cdot r_{B}+(1-2 m) r_{M}} \\
& \times M_{\star}^{-m+4 m \cdot m_{B}+(1-2 m) m_{\dot{M}}} \frac{\Omega_{\star}}{\left(1+\frac{f^{2}}{K^{2}}\right)^{m}} .
\end{aligned}
$$

From this generic formulation, it is now possible to constrain the different exponents by several observational trends into account.

\section{Observational constraints}

\subsection{Relationships between stellar parameters}

Some observational trends are based on a set of main-sequence stars in the unsaturated rotation regime (for which the Rossby number is greater than a certain threshold) and, therefore, they take scaling laws between stellar parameters into account, depending on a specific stellar evolution model. In particular, we need to consider mass-radius and mass-luminosity relationships during the main sequence. Here, we assume the general correlations

$L_{\star} \propto M_{\star}^{\eta}$

$R_{\star} \propto M_{\star}^{\xi}$

where $\eta$ and $\xi$ are constants that depend on which stellar model is being considered. Therefore, the upcoming formulations, which are only valid during the main sequence, can accommodate any evolutionary model. As a default, we use the typical values $\eta=4$ and $\xi=0.9$ (Kippenhahn \& Weigert 1994).

\subsection{Constraints form stellar rotational evolution}

As already introduced, stellar spin down studies in open clusters, which were first performed on the Pleiades, Ursa Major, and the Hyades, show that the rotation rate of evolved main-sequence stars tends to converge at the solar rate on a sequence where $\Omega_{\star} \propto t^{-1 / 2}$ (Skumanich 1972). By assuming that the moment of inertia of the star is constant, the wind braking torque is constrained in the unsaturated regime to scale as the cube of the stellar rotation rate

$\Gamma_{\text {wind }} \propto \Omega_{\star}^{3}$.

In the following sections, we assume that gyrochonology is valid thanks to the Skumanich law. This way, if a decrease of the wind braking efficiency is genuinely occurring for evolved stars (van Saders et al. 2016), then we only consider solar-type stars that are younger than the Sun to ensure the validity of Eq. (14). A stalling of the magnetic braking could be modeled in our formalism with a "resaturation" regime where the Rossby number is greater than a certain threshold $\mathrm{Ro}_{\text {break }}$.

Matt et al. (2015) studied the different dependencies of the wind braking torque in more detail to explain some characteristic features of the distribution of stellar rotation periods in open clusters and Kepler stars as a function of their mass. To this end, they focus on two kinds of stellar populations: the slow rotators in an unsaturated regime and the fast saturated rotators. The saturation threshold is given by a value of the Rossby number $\mathrm{Ro}_{\mathrm{sat}}$, 
which is assumed to be independent of any stellar parameters, at least at zeroth-order. To explain the mass dependency of the stellar spin down, they took the Skumanich law in the unsaturated regime and a linear saturation for the wind braking torque into account, leading to the following prescription

$\Gamma_{\text {wind }}=\Gamma_{\odot}\left(\frac{R_{\star}}{R_{\odot}}\right)^{a}\left(\frac{M_{\star}}{M_{\odot}}\right)^{b}\left(\frac{\mathrm{Ro}}{\mathrm{Ro}_{\odot}}\right)^{-2}\left(\frac{\Omega_{\star}}{\Omega_{\odot}}\right) \quad$ (unsaturated)
$\Gamma_{\text {wind }}=\Gamma_{\odot}\left(\frac{R_{\star}}{R_{\odot}}\right)^{a}\left(\frac{M_{\star}}{M_{\odot}}\right)^{b}\left(\frac{\mathrm{Ro}_{\text {sat }}}{\mathrm{Ro}_{\odot}}\right)^{-2}\left(\frac{\Omega_{\star}}{\Omega_{\odot}}\right) \quad$ (saturated),

with $\Gamma_{\odot}=6.3 \times 10^{30} \mathrm{erg}, a=3.1$, and $b=0.5$.

This prescription can be compared with our formulation, in Eq. (11), by assuming $f \ll 1$ and by neglecting secular changes of the stellar parameters. It is thus possible to link the magnetic field of the star and the mass loss through the following conditions

$p_{B}=\frac{1}{2 m}-\frac{1-2 m}{4 m} p_{\dot{M}} \quad$ (unsaturated)

$p_{B}=-\frac{1-2 m}{4 m} p_{\dot{M}} \quad$ (saturated)

$r_{B}=\frac{a-(2+5 m)}{4 m}-\frac{1-2 m}{4 m} r_{\dot{M}}$

$m_{B}=\frac{b+m}{4 m}-\frac{1-2 m}{4 m} m_{\dot{M}}$.

Equation (17) is similar to the condition obtained by Skumanich (2019) if we use $p_{B}=1 / \beta$ and $p_{\dot{M}}=\alpha / \beta$, according to his notation. However, no correlation between $B_{\star}$ and $\dot{M}$ is assumed here. The wind torque parametrization therefore gives us the opportunity to infer the mass loss prescription from the magnetic field prescription and reciprocally.

In Fig. 2 we illustrate the interdependencies in this first set of exponents. More precisely, the $p_{B}$ exponent is expressed as a function of $p_{\dot{M}}$ from Eq. (17) for a dipolar field (solid black line), a quadrupolar field (dashed black line) and an octupolar field (dotted black line) in the case of an unsaturated rotation regime. The dashed blue lines correspond to magnetic field prescriptions from See et al. (2017; S17) and Vidotto et al. (2014; V14). The blue crosses represent the magnetic field and mass loss prescriptions from Johnstone et al. (2015a), Tu et al. (2015) and Sadeghi Ardestani et al. (2017), resp. J15, T15, SA17. Those scaling laws were derived to reproduce rotation rates of open clusters and are in good agreement with a square root spin down law. As already pointed out by Skumanich (2019), a wide range of exponents is admissible from the different prescriptions considered.

The magnetic topology also has a significant influence on the $\dot{M}-B_{\star}$ prescriptions. Indeed, the value of the $m$ exponent decreases with an increasing complexity of the topology, corresponding to higher-order multipoles (Réville et al. 2015a). Here, we use $m=0.15$ as a quadrupolar field (see the dashed black line in Fig. 2) and $m=0.11$ as an octupolar field (see the dotted black line in Fig. 2). More complex magnetic fields lead to steeper slopes in Fig. 2 and, therefore, to a less constrained magnetic field. In regards to the mass loss, it tends to be proportional to $\mathrm{Ro}^{-2}$. Only one prescription of $B_{\star}$ and $\dot{M}$ is compatible with the Skumanich law for any magnetic topology (see the dark red dashed lines in Fig. 2) and corresponds to the one highlighted by Skumanich (2019). This configuration leads to a linear magnetic field-rotation relation and a quadratic mass loss-rotation relation. This way, such a prescription may be used as a first estimate of $B_{\star}$ and $\dot{M}$ based on spin down considerations without having

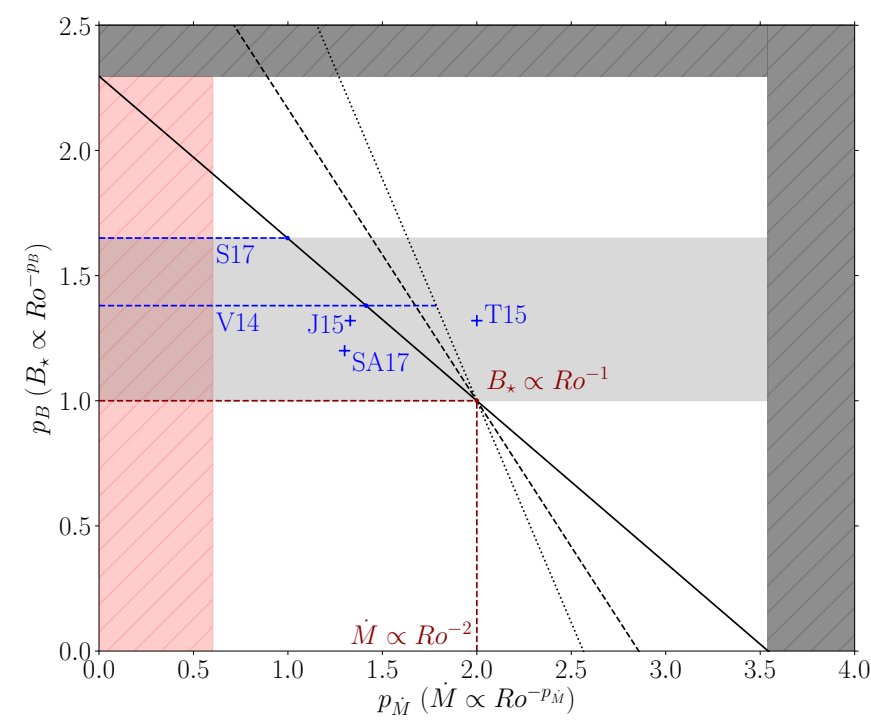

Fig. 2. Correlation $p_{B}-p_{\dot{M}}$ for a dipolar (solid black line), a quadrupolar (dashed black line), and an octupolar (dotted black line) topology in the unsaturated regime. Dashed blue lines: magnetic field prescriptions from See et al. (2017, S17) and Vidotto et al. (2014, V14). Blue crosses: magnetic field and mass loss prescriptions from Johnstone et al. (2015a, J15), Tu et al. (2015, T15), and Sadeghi Ardestani et al. (2017, SA17). In dark gray: excluded region in the dipolar case. Dashed dark red lines: prescriptions compatible with the Skumanich law for any magnetic topology. In light red: exponents corresponding to mass losses outside the envelope of Wood et al. (2005) data. The light gray band corresponds to the upper and lower bounds from Zeeman-Doppler imaging (ZDI) statistical studies (see Sect. 3.4 for more details).

to assume a particular magnetic topology. However, in the following sections, we use unspecified prescriptions for the magnetic field and the mass-loss rate in order to study the influence of additional observational constraints. It is important to note that complex fields can significantly modify the wind braking torque itself (Réville et al. 2015a; Garraffo et al. 2016). Furthermore, from those considerations, Garraffo et al. (2018) were able to reproduce the bimodal distribution of slow and fast rotators. Since the dipole component tends to dominate the wind braking torque for mixed geometries (Finley \& Matt 2018), such a topology is assumed in the following sections by default.

\subsection{Constraints on the mass-loss rate}

Given the one-to-one correspondence between the $B_{\star}$ and $\dot{M}$ exponents, constraints on the mass loss affect the magnetic field and reciprocally. First, the mass loss is enhanced by stellar rotation (Wood et al. 2005; Suzuki et al. 2013; Holzwarth \& Jardine 2007), leading to $p_{\dot{M}} \geq 0$. Therefore, we obtain

$p_{B} \leq \frac{1}{2 m} \approx 2.3 \quad$ (unsaturated)

$p_{B} \leq 0 \quad$ (saturated).

Furthermore, Wood et al. (2002, 2005) showed a correlation between the mass loss and the X-ray stellar flux $F_{\mathrm{X}}$ for unsaturated main-sequence stars, which is expressed as

$\dot{M} \propto R_{\star}^{2} F_{\mathrm{X}}^{w}$,

where $w$ is a constant between $w_{\min } \approx 0.3$ and $w_{\max } \approx 1.9$, which is consistent with the obervations from Wood et al. (2005). To convert this correlation into a $\dot{M}\left(\mathrm{Ro}, R_{\star}, M_{\star}\right)$ prescription, we 
consider a relationship between the coronal activity and the stellar rotation for unsaturated main-sequence stars:

$\frac{L_{\mathrm{X}}}{L_{\star}} \propto \mathrm{Ro}^{-p_{\mathrm{L}}}$

where $L_{\mathrm{X}}$ is the X-ray stellar luminosity, $L_{\star}$ is the luminosity of the star, and $p_{\mathrm{L}}$ is an exponent between $p_{\mathrm{L}, \min }=2$ and $p_{\mathrm{L}, \max }=3$ (Pizzolato et al. 2003; Wright et al. 2011; Reiners et al. 2014). If we take the scaling laws from Sect. 3.1 into account, it is possible to estimate the mass loss as a function of the Rossby number and the stellar mass from stellar activity considerations. Indeed, the $\mathrm{X}$-ray flux becomes

$F_{\mathrm{X}} \propto \mathrm{Ro}^{-p_{\mathrm{L}}} M_{\star}^{\eta-2 \xi}$,

which leads to the following mass loss:

$\dot{M} \propto \mathrm{Ro}^{-p_{\mathrm{L}} w} M_{\star}^{(\eta-2 \xi) w+2 \xi}$.

For identification purposes, the exponents of $\dot{M}$ can be inferred from the set $\left\{\eta, \xi, p_{\mathrm{L}}, w\right\}$ :

$p_{\dot{M}}=p_{\mathrm{L}} w$

$\xi r_{\dot{M}}+m_{\dot{M}}=(\eta-2 \xi) w+2 \xi$.

To be consistent with the data from Wood et al. (2005), the most flexible constraint on the $p_{\dot{M}}$ exponent can be obtained from Eq. (27) as

$0.6 \leq p_{\dot{M}} \leq 5.7$.

According to Eq. (29), the values of $p_{\dot{M}}$, leading to $\left(F_{\mathrm{X}}, \dot{M}\right)$ outside the envelope of the data from Wood et al. (2005), define the exclusion red regions in Figs. 2 and 3. Equations (27) and (28) then give an additional constraint on the different exponents of the mass loss prescription

$\xi r_{\dot{M}}+m_{\dot{M}}-2 \xi=\frac{\eta-2 \xi}{p_{\mathrm{L}}} p_{\dot{M}}$

This alone dictates the mass and radius dependency of the stellar magnetic field, which is easier to observe, through Eqs. (17)(20), such that

$4 m\left(\xi r_{B}+m_{B}\right)=\xi(a-m-4)+b+m-\frac{\eta-2 \xi}{p_{\mathrm{L}}}\left(2-4 m p_{B}\right)$.

Equation (31) shows that the mass-radius dependency of the magnetic field can be inferred from its Rossby number dependency, as shown in Fig. 3, where the green area represents the exponents of the magnetic field prescription, which is compatible with the different constraints we considered. The negative values of $\xi r_{B}+m_{B}$ show that the magnetic field should decrease with the stellar mass, which seems to be in agreement with dynamo models and observations (Johns-Krull \& Valenti 2000; Brun et al. 2015).

One can also notice in Fig. 3 that a stronger Rossby number dependency, for high values of $p_{B}$, leads to a weaker but non negligible dependency on stellar mass through the $\dot{M}-F_{\mathrm{X}}$ correlation. Futhermore, by considering the different exclusion regions (see the hatched areas in Fig. 3), we can infer that a magnetic field, which is solely a function of the Rossby number (i.e., $r_{B}=m_{B}=0$ ), cannot be consistent with the observed rotational dependency of $L_{\mathrm{X}}$ and $\dot{M}$ as well as with the $\dot{M}-F_{\mathrm{X}}$ correlation. To fulfill this set of conditions, we need to add an explicit mass dependency to the stellar magnetic field.

Only a linear combination of $r_{B}$ and $m_{B}$ is constrained here because of the mass-radius relationship. However, it is possible to discriminate the mass and radius dependencies by considering the magnetic topology.

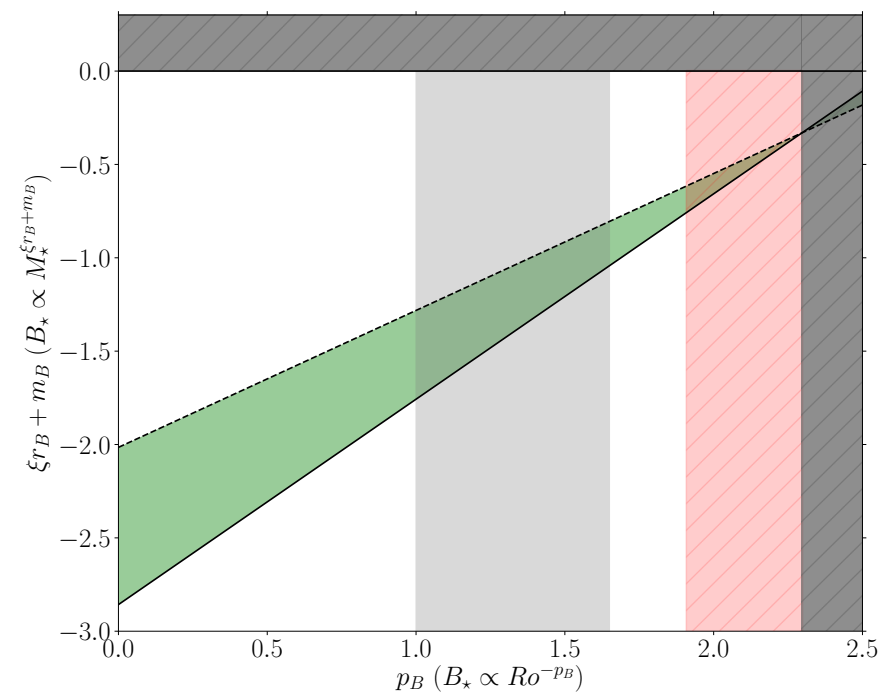

Fig. 3. Correlation between $p_{B}$ and $\xi r_{B}+m_{B}$ in the unsaturated regime for $p_{\mathrm{L}}=2$ (black line) and $p_{\mathrm{L}}=3$ (dotted). Here, $\eta=4$ and $\xi=$ 0.9. In dark gray: excluded region. In red: exponents corresponding to mass losses outside the envelope of Wood et al. (2005) data. In green: exponents consistent with the mass-loss constraints. The light gray band corresponds to the upper and lower bounds from ZDI statistical studies (see Sect. 3.4 for more details).

\subsection{Constraints on the magnetic field}

The stellar magnetic field can be constrained in a variety of ways. Spectropolarimetric studies have exhibited correlations between the large scale magnetic field and other stellar parameters. For example, in Vidotto et al. (2014), $B_{\star}$ is shown to scale as $\mathrm{Ro}^{-1.38 \pm 0.14}$, which leads to a mass loss of $p_{\dot{M}}=-1.41 \pm 0.22$. The X-ray luminosity of the star can also be considered to infer the magnetic field. Here, we assume a correlation of $L_{X}-B_{\star}$ such that (Pevtsov et al. 2003; Vidotto et al. 2014)

$\frac{L_{\mathrm{X}}}{L_{\star}} \propto B_{\star}^{v}$,

which leads to $p_{B}=p_{\mathrm{L}} / v$. For instance, in assuming that $p_{B}=$ $-1.38 \pm 0.14$ and $p_{\mathrm{L}}=2.5 \pm 0.5$, this leads to $v=1.81 \pm 0.6$, which can be compared with the value $v=1.61 \pm 0.15$, which was obtained in Vidotto et al. (2014) through a power-law fit.

Since the magnetic field is enhanced by the stellar rotation (Noyes et al. 1984; Brandenburg \& Saar 2000; Petit et al. 2008), the exponent $p_{B}$ has to be nonnegative, leading to the following constraint on the mass-loss rate in the unsaturated regime

$p_{\dot{M}} \leq \frac{2}{1-2 m} \approx 3.5 . \quad$ (unsaturated)

At saturation, with Eq. (22), such a condition gives $p_{B}=0$ and $p_{\dot{M}}=0$, which means a mass loss and a magnetic field are independent of the stellar rotation rate.

In the following sections, we consider lower and upper bounds for the values of $p_{B}$ and $p_{\dot{M}}$ in order to be consistent with observational trends. The measured stellar magnetic field from Zeeman broadening and Zeeman-Doppler Imaging (ZDI; see Montesinos \& Jordan 1993; Vidotto et al. 2014; See et al. 2017) have only exhibited linear or super-linear dependencies between the large-scale magnetic field and the Rossby number. In order to take this into account, we thus adopt $p_{B} \geq 1$. The See et al. (2017) prescription, that is, $p_{B}=1.65$, is used as an upper bound. The values of all of the exponents associated with these 
two scenarios are given in Table 1. It is a common knowledge that ZDI maps, representing the large-scale unsigned magnetic flux, do not give any information about the small-scale magnetic field, which might be dominant for young fast rotating stars. However, a correlation between measurements from ZeemanDoppler imaging and Zeeman broadening (See et al. 2019) gives some confidence in the general trends found in the literature.

The bounds we assume above are greater than the exponents predicted by some scaling laws for stellar dynamos $\left(p_{B}=0\right.$ for the equipartition, $p_{B}=1 / 4$ for the buoyancy regime, and $p_{B}=1 / 2$ for the magnetostrophy regime; for a review, see Augustson et al. 2017). This discrepancy likely comes from the fact that dynamo scaling laws and ZDI observations do not relate to the same magnetic field. The measured magnetic field from ZDI studies corresponds to the average unsigned photospheric flux $\left\langle B_{V}\right\rangle$, which is an estimate of the large scale magnetic field at the stellar surface. Dynamo-based scaling laws aim to estimate the stellar magnetic field over a wide spectral range. The latter can be linked to the average unsigned surface field strength, $\left\langle B_{I}\right\rangle$, obtained from Zeeman broadening, by means of a filling factor $f$ that represents the fraction of the stellar surface which is magnetized (see Reiners 2012 for more details). By exhibiting a correlation between $\left\langle B_{V}\right\rangle$ and $\left\langle B_{I}\right\rangle$, See et al. (2019) estimated a filling factor from a dynamo-produced magnetic field in the equipartition regime and show a strong Rossby number dependency in their $f$ estimate, which could be a means of explaining this difference.

In a nutshell, it is possible to rely on a wind torque parametrization to provide $B_{\star}$ and $\dot{M}$ estimates that are consistent with spin down and X-ray emission constraints (cf. Eqs. (17)-(20) and (31)), given for example the $B_{\star}-$ Ro relation (i.e., the $p_{B}$ exponent). In a quest to better understand the link between stellar and wind properties, we expand on our set of prescriptions to probe the coronal properties of the star by relying on a wind model.

\subsection{Constraints on the coronal properties}

\subsubsection{Probing the coronal properties: The role of magnetic topology}

As seen in the previous section, the mass loss behavior can be constrained by the stellar magnetic field and the wind braking torque. This way, the knowledge of the $\dot{M}$ expression allows us to go further by inferring prescriptions for the coronal temperature and density by means of the X-ray stellar emission. Indeed, their rotational dependency has often been constrained in the literature through the high-energy activity of the star (Mestel \& Spruit 1987; Ivanova \& Taam 2003; Holzwarth \& Jardine 2007).

However, X-ray emission and wind acceleration, at least for the fast wind, are believed to arise from different regions in the corona, that is, from dead zones and coronal holes, respectively. Therefore, here, we assume that the coronal temperature $T_{\mathrm{c}}$ and the base density $n_{\mathrm{c}}$ in the open-field regions are the quantities ruling the mass loss for a given wind model (cf. Eq. (10) for example), whereas the X-ray luminosity can be inferred from radiative losses by knowing the temperature $T_{1}$ and the density $n_{1}$ in closed-field regions (cf. Appendix B). In other words, the $\dot{M}$ prescription from the previous sections allows us to link $T_{\mathrm{c}}$ and $n_{\mathrm{c}}$, while the Rossby dependency of $L_{\mathrm{X}}$, which has to be consistent with Eq. (24), correlates $T_{1}$ and $n_{1}$. All of those connexions are summarized in Fig. 4. Since $T_{1}$ can be obtained from observations (Preibisch 1997; Johnstone \& Güdel 2015; Wood et al. $2018)$, we need an additional constraint to relate $\left(T_{\mathrm{c}}, n_{\mathrm{c}}\right)$ to $\left(T_{1}, n_{1}\right)$.

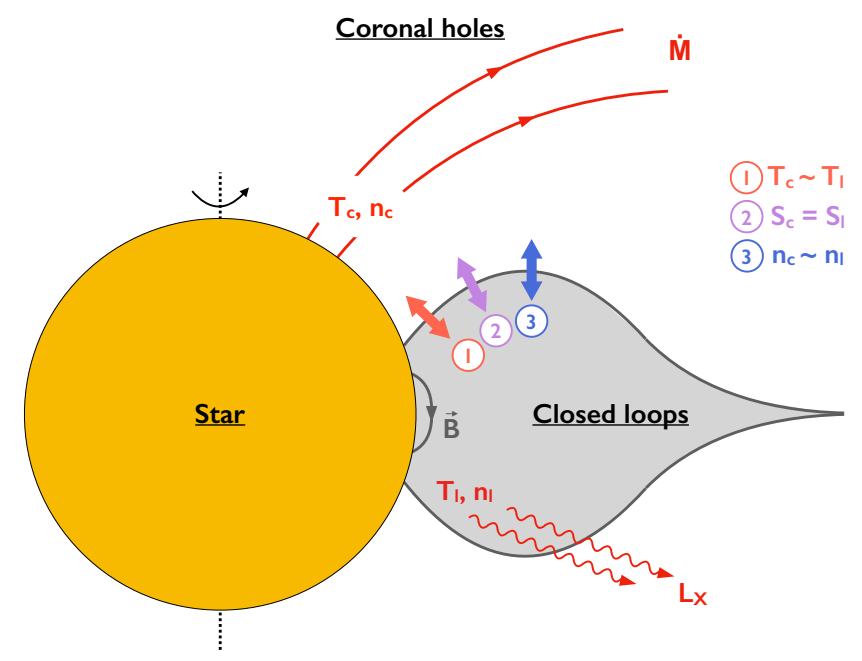

Fig. 4. Sketch of the coupling between the coronal temperature and density in the open-field and the closed-field regions, the X-ray luminosity, and the mass-loss rate. Several scenarios are considered to connect the closed loops to the coronal holes. Scenario 1, in red: single temperature scaling. Scenario 2, in purple: entropy equilibrium. Scenario 3, in blue: single density scaling.

To this end, we consider three scenarios to couple the openfield and the closed-field regions. In scenario 1 (in red in Fig. 4), we consider a common scaling law for $T_{\mathrm{c}}$ and $T_{1}$ (Johnstone et al. 2015b; Ó Fionnagain \& Vidotto 2018), which leads to different scalings for the densities. In scenario 2 (in purple in Fig. 4), we assume an entropy equilibrium between the closed loops and the coronal holes. In scenario 3 (in blue in Fig. 4), we assume a common scaling for $n_{\mathrm{c}}$ and $n_{1}$ (Ivanova \& Taam 2003; Holzwarth \& Jardine 2007; See et al. 2014), which leads to different scalings for the temperatures.

It is worth noticing that scenarios 1 and 3 lead to drastically different trends for the temperature $T_{\mathrm{c}}$. Therefore, a scenario where both the density and the temperature possess the same scaling in the open and closed field regions is unrealistic.

Such an approach allows us to deal with the local magnetic field distribution in an admittedly simplified way by distinguishing quiet open-field regions from closed loops associated with active regions. This distinction translates into different coronal temperature and density prescriptions, which are always chosen to be compatible with our derived wind massloss rate in the polytropic formalism. Of course, at the surface of the star, the plasma dynamics and heating mechanisms are much more involved than in our simplified approach (Wedemeyer-Böhm et al. 2009). Still this is a first step in characterizing the general properties of the coronae of cool stars, and we intend to consider more realistic modeling for the detailed coronal heating mechanism in the future.

In what follows, we see how to constrain the coronal temperatures and densities from $L_{\mathrm{X}}$ and $\dot{M}$ (Sects. 3.5.2 and 3.5.3, respectively). Then, we assess the different scenarios in Sects. 3.5.4-3.5.6.

\subsubsection{Coronal temperature and density: X-ray luminosity consistency}

The temperature and the density in closed loops are connected to the X-ray luminosity, which must be consistent with the prescriptions we adopt in Eq. (24). From radiative losses considerations, the X-ray luminosity can be expressed as (cf. Appendix B) 
$L_{\mathrm{X}} \propto\left(\frac{R_{\star}}{R_{\odot}}\right)^{3}\left(\frac{B_{\star}}{B_{\odot}}\right)\left(\frac{n_{1}}{n_{1, \odot}}\right)^{\frac{3}{2}}\left(\frac{T_{1}}{T_{1, \odot}}\right)^{-\frac{7}{6}}$

In order to standardize the different prescriptions, we consider a power-law expression for the temperature and the density in the dead zones, that is,

$$
\begin{aligned}
& T_{1} \propto\left(\frac{\mathrm{Ro}}{\mathrm{Ro}_{\odot}}\right)^{-p_{\mathrm{T}, \mathrm{l}}}\left(\frac{R_{\star}}{R_{\odot}}\right)^{r_{\mathrm{T}, 1}}\left(\frac{M_{\star}}{M_{\odot}}\right)^{m_{\mathrm{T}, 1}}, \\
& n_{1} \propto\left(\frac{\mathrm{Ro}}{\mathrm{Ro}_{\odot}}\right)^{-p_{\mathrm{n}, \mathrm{l}}}\left(\frac{R_{\star}}{R_{\odot}}\right)^{r_{\mathrm{n}, \mathrm{l}}}\left(\frac{M_{\star}}{M_{\odot}}\right)^{m_{\mathrm{n}, 1}} .
\end{aligned}
$$

Furthermore, for main-sequence stars in the unsaturated regime, we assume a correlation between the X-ray flux and the coronal temperature in the closed loops, such that (Preibisch 1997; Johnstone \& Güdel 2015; Wood et al. 2018)

$$
T_{1} \propto F_{\mathrm{X}}^{j_{1}}
$$

with an exponent $j_{1}=0.26$ (Johnstone \& Güdel 2015). Equation (25), with relationships from Sect. 3.1 , then result in a $T_{1}$ $\left(\right.$ Ro, $M_{\star}$ ) formulation

$T_{1} \propto \mathrm{Ro}^{-p_{\mathrm{L}} j_{1}} M_{\star}^{(\eta-2 \xi) j_{1}}$

This leads by identification to

$p_{\mathrm{T}, 1}=p_{\mathrm{L}} j_{1}$.

The rotational dependency of the density can therefore be deduced from the magnetic field prescription for a given set $\left\{p_{\mathrm{L}}, j_{1}\right\}$ by ensuring the consistency with Eq. (24):

$p_{\mathrm{n}, 1}=\frac{2}{3}\left(p_{\mathrm{L}}-p_{B}\right)+\frac{7}{9} p_{\mathrm{L}} j_{1}$

The X-ray luminosity therefore gives us the possibility to constrain the Rossby dependency of the temperature and the density in closed loops.

We now need to study the connection between the X-ray luminosity and the temperature in coronal holes, which will allow us to infer the mass-radius dependency of $T_{\mathrm{c}}$ from its rotational dependency, as we did for the mass-loss rate in Sect. 3.3. X-ray emission and wind acceleration have similar sources that are closely linked to the heating of the corona, which is probably due to the transport of energy from the photosphere through weakly dissipative Alfvén waves. These phenomena involve steep density gradients (Heyvaerts \& Priest 1983) and nonlinear interactions between inward and outward perturbations (Velli et al. 1989), among others (for a review, see Mathioudakis et al. 2013; Cranmer et al. 2017). While this process is efficient in closed loops thanks to the magnetic topology, it requires wave reflections, for example through the parametric instabilities in coronal holes (Réville et al. 2018), thus reducing the heating efficiency in those regions. To take this behavior into account, we assume a correlation between the X-ray flux and the coronal temperature $T_{\mathrm{c}}$, such as in the closed loops case, with an unspecified exponent that is different from $j_{1}$ because of the possible difference of heating efficiency between open-field and closed-field regions.

\subsubsection{Coronal temperature and density: Polytropic wind model considerations}

The temperature and the density in coronal holes need to be consistent with our mass loss prescription, which is constrained in Sects. 3.1-3.4. We consider the following power-law expression for the coronal temperature:

$T_{\mathrm{c}} \propto\left(\frac{\mathrm{Ro}}{\mathrm{Ro}_{\odot}}\right)^{-p_{\mathrm{T}}}\left(\frac{R_{\star}}{R_{\odot}}\right)^{r_{\mathrm{T}}}\left(\frac{M_{\star}}{M_{\odot}}\right)^{m_{\mathrm{T}}}$.

Such a prescription has to fulfill the condition that $\dot{M}>0$, which defines a maximal value for the $p_{\mathrm{T}}$ exponent (see Appendix $\mathrm{C}$ for more details).

In knowing the value of $T_{\mathrm{c}}$ for a given wind model, it is then possible to infer the coronal density to obtain a consistent massloss rate. As we see in Sect. 2.4 with the Eq. (10), the mass loss can be expressed as a function of stellar parameters and coronal properties. To be consistent with the power-law prescription from Eq. (9), the coronal density $n_{\mathrm{c}}$ has to be expressed as

$n_{\mathrm{c}} \propto \mathrm{Ro}^{-p_{\dot{M}}} R_{\star}^{r_{\dot{M}}} M_{\star}^{m_{\dot{M}}-2} T_{\mathrm{c}}^{\frac{3}{2}}\left[1-\frac{T_{\min , \odot}}{T_{\odot}} \frac{M_{\star}}{M_{\odot}} \frac{R_{\odot}}{R_{\star}} \frac{T_{\odot}}{T_{\mathrm{c}}}\right]^{\frac{3 \gamma-5}{2(\gamma-1)}}$.

It is important to keep in mind that this equation is valid if $c_{\mathrm{s}} / v_{\text {esc }} \ll 1$, where $c_{\mathrm{s}}$ is the speed of sound in the stellar corona and $v_{\text {esc }}$ is the escape velocity at the stellar surface (cf. Appendix A). In light of this condition, high coronal temperatures could invalidate this analytical expression of $n_{\mathrm{c}}$.

If we assume that the coronal temperature varies slightly for the stellar parameters we consider, we can approximate this expression to a power law on the main sequence, such that (see details in Appendix D)

$n_{\mathrm{c}} \propto\left(\frac{\mathrm{Ro}}{\mathrm{Ro}_{\odot}}\right)^{-p_{\mathrm{n}}}\left(\frac{R_{\star}}{R_{\odot}}\right)^{r_{\mathrm{n}}}\left(\frac{M_{\star}}{M_{\odot}}\right)^{m_{\mathrm{n}}}$,

with:

$p_{\mathrm{n}}=p_{\dot{M}}+\left(\frac{3}{2}-F(\gamma)\right) p_{\mathrm{T}}$,

$r_{\mathrm{n}}=r_{\dot{M}}+\frac{3}{2} r_{\mathrm{T}}-F(\gamma)\left(1+r_{\mathrm{T}}\right)$

$m_{\mathrm{n}}=m_{\dot{M}}-2+\frac{3}{2} m_{\mathrm{T}}-F(\gamma)\left(m_{\mathrm{T}}-1\right)$,

and $F(\gamma)=\frac{5-3 \gamma}{2(\gamma-1)} \frac{T_{\min , \odot} / T_{\odot}}{1-T_{\min , \odot} / T_{\odot}}$.

Therefore, for a given mass-loss rate prescription, a oneto-one correspondence between $\left\{p_{\mathrm{T}}, r_{\mathrm{T}}, m_{\mathrm{T}}\right\}$ and $\left\{p_{\mathrm{n}}, r_{\mathrm{n}}, m_{\mathrm{n}}\right\}$ occurs by considering a pressure-driven polytropic wind. Furthermore, we are able to infer $\xi r_{\mathrm{T}}+m_{\mathrm{T}}$ from the $p_{\mathrm{T}}$ exponent thanks to a $T_{\mathrm{c}}-F_{\mathrm{X}}$ correlation (cf. Appendix C). Given that the Rossby dependency of $T_{1}$ and $n_{1}$ is already known through Eqs. (39) and (40), respectively, we only need one additional constraint to fully determine the expression of $T_{\mathrm{c}}$ and $n_{\mathrm{c}}$. Here, we chose to connect the rotational dependency of the temperature and the density in open and closed regions by means of the three scenarios we present in Sect. 3.5.1. Now, we detail the implications of these scenarios in Sects. 3.5.4-3.5.6.

\subsubsection{Scenario 1: Single temperature scaling}

To connect closed loops to coronal holes, we can adopt a single scaling law for the temperature (i.e., $T_{1} \propto T_{\mathrm{c}}$ ), leading to 
the same X-ray flux-temperature correlation in both regions. This way, the Johnstone \& Güdel (2015) prescription, coupled with the $L_{X}-$ Ro relation, and the results from Sect. 3.1 provide a complete expression for $T_{\mathrm{c}}$. One cannot assume weak variations of the coronal temperature, which means that a power-law expression for $n_{\mathrm{c}}$ may be a loose approximation in this scenario (cf. Appendix D). However, the coronal density can be directly inferred from our wind model through Eq. (42) in order to keep a consistent mass loss. Those considerations then allow us to estimate $T_{\mathrm{c}}$ and $n_{\mathrm{c}}$ by means of the following prescriptions

$p_{\mathrm{T}}=p_{\mathrm{L}} j_{1}$,

$\xi r_{\mathrm{T}}+m_{\mathrm{T}}=\frac{\eta-2 \xi}{p_{\mathrm{L}}} p_{\mathrm{T}}$

$n_{\mathrm{c}} \propto \mathrm{Ro}^{-p_{M}} R_{\star}^{r_{\dot{M}}} M_{\star}^{m_{\dot{M}}-2} T_{\mathrm{c}}^{\frac{3}{2}}\left[1-\frac{T_{\min , \odot}}{T_{\mathrm{c}}} \frac{M_{\star}}{M_{\odot}} \frac{R_{\odot}}{R_{\star}}\right]^{\frac{3 \gamma-5}{2(\gamma-1)}}$

In a saturated rotation regime, it is impossible from Eq. (50) to keep a constant value for both $T_{\mathrm{c}}$ and $n_{\mathrm{c}}$. For simplicity purposes, we assume a single temperature at saturation, the corresponding coronal density being inferred from the $\dot{M}$ prescription.

The robustness of the single temperature scaling hypothesis can, nevertheless, be questioned. In closed loops, available estimates of $T_{1}$ (Preibisch 1997; Johnstone \& Güdel 2015; Wood et al. 2018) fall back on an emission measure weighted average coronal temperature, based on heavy ions emission (Güdel 2007). From the wind model point of view, which is based on the modeling of a perfectly ionized hydrogen gas, the electron temperature, which is similar to the proton temperature in this context, is required to compute the mass-loss rate. Thus, a single scaling law for both temperatures may appear as a loose assumption. Indeed, if different radial profiles of temperature are observed in the solar wind for these two populations (Cranmer et al. 2017), with a higher temperature for heavy ions, their dependency on stellar parameters is still unknown.

Furthermore, if we assume $T_{\mathrm{c}} \propto T_{1}$, slower rotators may have a colder corona, leading to extremely strong densities to keep a consistent mass loss. For example, for a solar twin with a rotation period of 56 days, the single temperature scaling scenario leads to $n_{\mathrm{c}} \approx 19.58 n_{\odot}$, which has to be compared to $n_{1} \approx 0.48 n_{\odot}$, according to Eq. (40). Coronal holes of slow rotators would be abnormally dense, which may suggest that a single scaling law for the temperature might be inconsistent with the other hypothesis of our formalism, especially the choice of our wind model. Because of those points, we consider other scenarios in what follows.

\subsubsection{Scenario 2: Entropy equilibrium}

A second possibility is to assume entropy equilibrium between closed and open field regions, allowing both density and temperature to vary simultaneously. This allows us to derive the following relation:

$\ln T_{1}-\left(\gamma_{\mathrm{ad}}-1\right) \ln n_{\mathrm{l}}=\ln T_{\mathrm{c}}-\left(\gamma_{\mathrm{ad}}-1\right) \ln n_{\mathrm{c}}$,

where $\gamma_{\mathrm{ad}}=c_{\mathrm{p}} / c_{v}=5 / 3$ is the standard adiabatic exponent for an ideal gas. If we assume a power-law expression for $n_{\mathrm{c}}$ as in Eq. (43), this balance then gives the temperatures and densities for the Rossby dependency as follows:

$p_{\mathrm{T}, 1}-\left(\gamma_{\mathrm{ad}}-1\right) p_{\mathrm{n}, 1}=p_{\mathrm{T}}-\left(\gamma_{\mathrm{ad}}-1\right) p_{\mathrm{n}}$.

Along with Eq. (44), which dictates the rotational dependency of the mass-loss rate in our wind model, one can express the $p_{\mathrm{T}}$ and $p_{\mathrm{n}}$ exponents as follows:

$$
\begin{aligned}
& p_{\mathrm{T}}=\frac{\left(\gamma_{\mathrm{ad}}-1\right)\left(p_{\mathrm{n}, 1}-p_{\dot{M}}\right)-p_{\mathrm{L} j_{1}}}{\left(\gamma_{\mathrm{ad}}-1\right)[3 / 2-F(\gamma)]-1} \\
& p_{\mathrm{n}}=p_{\dot{M}}+\left(\frac{3}{2}-F(\gamma)\right) p_{\mathrm{T}}
\end{aligned}
$$

The $T_{\mathrm{c}}-F_{\mathrm{X}}$ correlation (cf. Appendix C), along with the wind model through Eqs. (45) and (46), dictate the mass-radius dependency of $T_{\mathrm{c}}$ and $n_{\mathrm{c}}$ as

$\xi r_{\mathrm{T}}+m_{\mathrm{T}}=\frac{\eta-2 \xi}{p_{\mathrm{L}}} p_{\mathrm{T}}$
$\xi r_{\mathrm{n}}+m_{\mathrm{n}}=\xi r_{\dot{M}}+m_{\dot{M}}-2+\left(\xi r_{\mathrm{T}}+m_{\mathrm{T}}\right)\left[\frac{3}{2}-F(\gamma)\right]+(1-\xi) F(\gamma)$.

Equations (53)-(56) then define the power-law expressions of $T_{\mathrm{c}}$ and $n_{\mathrm{c}}$ for this scenario.

At high rotation rates, as in Sect. 3.3, we see that the linear saturation of the wind braking torque leads to a magnetic field and a mass loss that is independent of the Rossby number, that is, $p_{B}=p_{\dot{M}}=0$. Similar behavior has been observed for the X-ray activity of stars (Pizzolato et al. 2003; Wright et al. 2011). Here, we assume for simplicity that $p_{\mathrm{L}}=0$ in the saturated regime. By relying on calculations that are similar to those presented in Sects. 3.5.2 and 3.5.3, we can show that $p_{\mathrm{n}}=p_{\mathrm{T}}=0$ is an acceptable choice if we assume a similar behavior in closed loops.

\subsubsection{Scenario 3: Single density scaling}

Let us finally consider that the coronal density has a similar behavior in the closed-field and open-field regions. This way, two different scaling laws arise for the temperatures in closedfield or open-field regions. In this case, the coronal density $n_{\mathrm{c}}$, which launches the wind in the open-field regions, is proportional to the density in closed loops $n_{1}$. Therefore, it can be expressed as a power law with a Rossby exponent given by Eq. (40) to ensure a consistent X-ray luminosity. Assuming a power-law expression for $n_{\mathrm{c}}$ is a reasonable hypothesis if the temperature in coronal holes only presents small variations with respect to stellar parameters (cf. Appendix D). Such a supposition seems to be consistent with Suzuki et al. (2013), who performed simulations of flux tubes that were heated by Alfvén wave dissipation in coronal holes. Indeed, they predict a weak dependency of the coronal temperature on the stellar magnetic field, which can be extrapolated to more fundamental stellar parameters through a dynamo relationship (see Eq. (7)). We can now assess the Rossby-dependency of the coronal temperature (i.e., the $p_{\mathrm{T}}$ exponent) through Eq. (44). All of those aspects allow us to determine the rotational dependency of $T_{\mathrm{c}}$ and $n_{\mathrm{c}}$ thanks to the following prescriptions

$$
\begin{aligned}
& p_{\mathrm{n}}=\frac{2}{3}\left(p_{\mathrm{L}}-p_{B}\right)+\frac{7}{9} p_{\mathrm{L} j_{1}}, \\
& p_{\mathrm{T}}=\frac{p_{\mathrm{n}}-p_{\dot{M}}}{\frac{3}{2}-F(\gamma)} .
\end{aligned}
$$

As for the entropy equilibrium scenario, the mass-radius dependency of $T_{\mathrm{c}}$ and $n_{\mathrm{c}}$ is determined through the wind model 


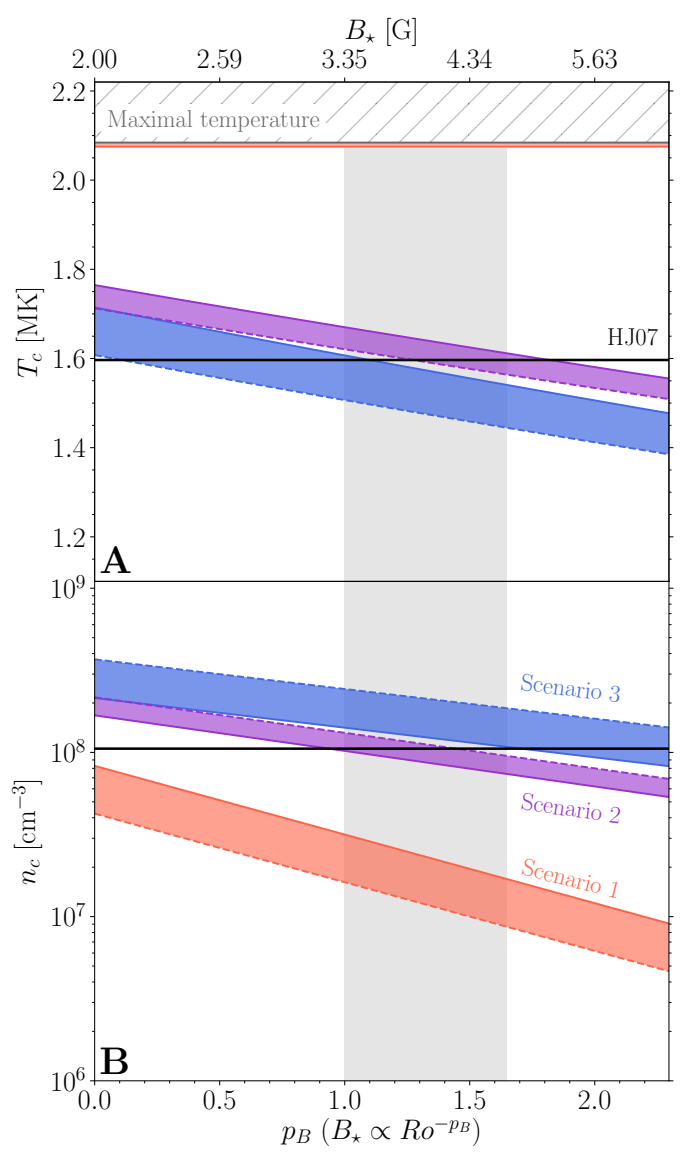

Fig. 5. Evolution of $T_{\mathrm{c}}$ (panel $A$ ) and $n_{\mathrm{c}}$ (panel $B$ ) as a function of $p_{B}$ for a solar twin with a rotation period of 15 days. Solid line: $p_{\mathrm{L}}=2$. Dashed lines: $p_{\mathrm{L}}=3$. In black: Holzwarth \& Jardine (2007) prescription. In light red: single temperature scaling scenario. In purple: entropy equilibrium scenario. In blue: single density scaling scenario. In hatched gray: values of coronal temperatures leading to $\dot{M}<0$. The light gray band corresponds to the upper and lower bounds of $p_{B}$, which we consider to be consistent with ZDI statistical studies.

and the $T_{\mathrm{c}}-F_{\mathrm{X}}$ correlation (cf. Appendix C):

$\xi r_{\mathrm{T}}+m_{\mathrm{T}}=\frac{\eta-2 \xi}{p_{\mathrm{L}}} p_{\mathrm{T}}$,

$\xi r_{\mathrm{n}}+m_{\mathrm{n}}=\xi r_{\dot{M}}+m_{\dot{M}}-2+\left(\xi r_{\mathrm{T}}+m_{\mathrm{T}}\right)\left[\frac{3}{2}-F(\gamma)\right]+(1-\xi) F(\gamma)$.

Equations (57)-(60) therefore give us the possibility to estimate the coronal temperature and density in open regions. As in Sect. 3.5.5, the assumption of a coronal temperature and density, independent of the Rossby number in the saturated rotation regime, is consistent with the different observational constraints we imposed.

Figure 5 shows the $T_{\mathrm{c}}$ and $n_{\mathrm{c}}$ estimates for a solar twin with a rotation period of 15 days (corresponding to Ro $=0.6$ in the Sadeghi Ardestani et al. 2017 prescription) in the three physical scenarios we analyzed. We set $\eta=4, \xi=0.9$ (Kippenhahn \& Weigert 1994), $\gamma=1.05$, and $T_{\odot}=1.5 \mathrm{MK}$ (Réville et al. 2016). One can notice that a stronger dependency of the magnetic field on the Rossby number, that is, higher values of $p_{B}$, leads to weaker values of $T_{\mathrm{c}}$ and $n_{\mathrm{c}}$. However, this is apart from the coronal temperature in Scenario 1, which remains constant (see the light red band in panel A of Fig. 5). Furthermore, coronal temperatures obtained in scenarios 2 and 3 (see the blue and purple bands in panel A of Fig. 5) only vary a little around the solar value, which is consistent with the assumptions we made to express $n_{\mathrm{c}}$ as a power law. Since the star we consider here is a solar twin that rotates more rapidly (i.e., with $\left.\mathrm{Ro} / \mathrm{Ro}_{\odot}<1\right)$, the positiveness criterion of the mass-loss rate (cf. Appendix C) sets a maximal coronal temperature (see the dark gray line in panel A of Fig. 5). From this condition, one can affirm that scenarios 2 and 3 are compatible with a transsonic wind. However, in scenario 1, the condition that $\dot{M}>0$ is only ensured for $p_{\mathrm{L}} \approx 2$; the coronal temperature is too high otherwise given a reasonable choice of $\left(\gamma, T_{\odot}\right)$.

Considering our lower and upper bounds for $p_{B}$ as described in Sect. 3.4, single density scaling and entropy equilibrium lead to coronal temperatures close to the Holzwarth \& Jardine (2007) prescription (see the black horizontal line in Fig. 5). Scenario 3 leads to temperatures that are weaker than the predicted value of Holzwarth \& Jardine (2007), resulting in slightly higher densities for a given mass-loss rate (see the blue band in panel B of Fig. 5). On the contrary, marginally higher temperatures and lower densities can be observed in scenario 2 (see the purple band in panel B of Fig. 5). Scenario 3, with a $T_{\mathrm{c}}$ prescription that is independent of the magnetic field and the mass-loss scaling laws, results in the highest values of the coronal temperature and the lowest values of the coronal density shown in Fig. 5 (in light red in Fig. 5). In light of those different aspects, scenarios 2 and 3 seem more likely to account for a consistent stellar spin down with a polytropic pressure-driven wind.

\section{Scaling laws and observations of individual systems}

\subsection{Using our scaling laws: A practical guide}

\subsubsection{Rossby number and rotation regime}

To deal with individual systems, we need to know their Rossby number to rely on our prescriptions. For the sake of simplicity, we use the stellar Rossby number here (cf. Eq. (1)) and more precisely the Sadeghi Ardestani et al. (2017) prescription for the convective turnover time. Following their approach, this characteristic time is defined as the ratio between the pressure scale height and the convective velocity that is estimated with the mixing length theory. They computed the relevant quantities at half a pressure scale height over the base of the convective zone and followed their evolution by means of the CESAM stellar evolution code (Morel \& Lebreton 2008). The formulation they obtained has the advantage of being valid during the premain sequence and the main sequence for metallicities ranging from $[\mathrm{Fe} / \mathrm{H}]=-0.5$ to 0.5 by falling back on the stellar convective mass as the control parameter. Given the age of the system we consider, such an expression can be simplified for mainsequence stars in order to obtain a power law that only depends on the stellar mass and the stellar radius, which leads to the following formulation for the convective turnover time:

$\tau_{\mathrm{c}} \propto M_{\star}^{-1} R_{\star}^{-1.2}$.

This prescription leads to a solar value of $\mathrm{Ro}_{\odot}=1.113$ and a saturation value of $\mathrm{Ro}_{\mathrm{sat}}=0.09$.

\subsubsection{Scaling laws: Numerical values of the exponents}

In the previous sections, we have been able to constrain the magnetic field, the mass loss, the coronal temperature, and the coronal density from wind braking considerations by assuming the 
Table 1. Parameters defining the $\Gamma_{\text {wind }}, B_{\star}, \dot{M}, T_{\mathrm{c}}$, and $n_{\mathrm{c}}$ prescriptions for $p_{B}=1$ and $p_{B}=1.65$ in the single density scaling scenario.

\begin{tabular}{|c|c|c|}
\hline Lower bound & Upper bound & Equation \\
\hline $\begin{array}{l}\text { Free parameters } \\
\gamma=1.05^{(a)} \\
m=0.2177^{(b)}\end{array}$ & $\begin{array}{l}- \\
- \\
\end{array}$ & $\begin{array}{c}(10) \\
(6)\end{array}$ \\
\hline $\begin{array}{l}\text { Constrained parameters } \\
T_{\odot}=1.5 \mathrm{MK}^{(a)} \\
\eta=4^{(c)}, \xi=0.9^{(c)} \\
p_{\mathrm{L}}=2^{(d)}\end{array}$ & $\begin{array}{l}- \\
- \\
-\end{array}$ & $\begin{array}{c}(10) \\
(12),(13) \\
(24)\end{array}$ \\
\hline$a=3.1^{(e)}, b=0.5^{(e)}$ & - & (15) \\
\hline $\begin{array}{l}p_{B}=1 \\
\xi r_{B}+m_{B}=-1.76\end{array}$ & $\begin{array}{l}p_{B}=1.65^{(f)} \\
\xi r_{B}+m_{B}=-1.04\end{array}$ & $\begin{array}{l}(7) \\
(7)\end{array}$ \\
\hline $\begin{array}{l}p_{\dot{M}}=2 \\
\xi r_{\dot{M}}+m_{\dot{M}}=4 \\
w=1\end{array}$ & $\begin{array}{l}p_{\dot{M}}=1 \\
\xi r_{\dot{M}}+m_{\dot{M}}=2.9 \\
w=0.5\end{array}$ & $\begin{array}{l}(9) \\
(9) \\
(23)\end{array}$ \\
\hline $\begin{array}{l}p_{\mathrm{T}}=0.11 \\
\xi r_{\mathrm{T}}+m_{\mathrm{T}}=0.12 \\
j=0.055\end{array}$ & $\begin{array}{l}p_{\mathrm{T}}=0.04 \\
\xi r_{\mathrm{T}}+m_{\mathrm{T}}=0.05 \\
j=0.02\end{array}$ & $\begin{array}{l}(58) \\
(59) \\
(\mathrm{C} .1)\end{array}$ \\
\hline $\begin{array}{l}p_{\mathrm{n}}=1.07 \\
\xi r_{\mathrm{n}}+m_{\mathrm{n}}=1.97\end{array}$ & $\begin{array}{l}p_{\mathrm{n}}=0.64 \\
\xi r_{\mathrm{n}}+m_{\mathrm{n}}=1.49\end{array}$ & $\begin{array}{l}(57) \\
(60) \\
\end{array}$ \\
\hline
\end{tabular}

References. $\quad{ }^{(a)}$ Réville et al. $\quad$ (2015b). $\quad{ }^{(b)}$ Matt et al. $\quad$ (2012). ${ }^{(c)}$ Kippenhahn \& Weigert (1994). ${ }^{(d)}$ Pizzolato et al. (2003). ${ }^{\left({ }^{e}\right)}$ Matt et al. (2015). ${ }^{(f)}$ See et al. (2017).

following prescriptions (at least in the entropy equilibrium and the single density scaling scenarios):

$$
\begin{aligned}
& \Gamma_{\text {wind }} \propto \dot{M}^{1-2 m} B_{\star}^{4 m} R_{\star}^{2+5 m} M_{\star}^{-m} \Omega_{\star}\left[1+\frac{f^{2}}{K^{2}}\right]^{-m} \\
& B_{\star} \propto \operatorname{Ro}^{-p_{B}}\left(\frac{R_{\star}}{R_{\odot}}\right)^{r_{B}}\left(\frac{M_{\star}}{M_{\odot}}\right)^{m_{B}} \\
& \dot{M} \propto \mathrm{Ro}^{-p_{\dot{M}}}\left(\frac{R_{\star}}{R_{\odot}}\right)^{r_{\dot{M}}}\left(\frac{M_{\star}}{M_{\odot}}\right)^{m_{\dot{M}}} \\
& T_{\mathrm{c}} \propto \mathrm{Ro}^{-p_{\mathrm{T}}}\left(\frac{R_{\star}}{R_{\odot}}\right)^{r_{\mathrm{T}}}\left(\frac{M_{\star}}{M_{\odot}}\right)^{m_{\mathrm{T}}} \\
& n_{\mathrm{c}} \propto \mathrm{Ro}^{-p_{\mathrm{n}}}\left(\frac{R_{\star}}{R_{\odot}}\right)^{r_{\mathrm{n}}}\left(\frac{M_{\star}}{M_{\odot}}\right)^{m_{\mathrm{n}}} .
\end{aligned}
$$

For example, the values for all of the exponents that are linked to the lower and upper bounds we considered in Sect. 3.4 (namely $1 \leq p_{B} \leq 1.65$ ) are given in Table 1 for the single density scaling scenario (in blue in Fig. 4). The corresponding exponents for the two other cases can be found in Appendix E. One can notice that the configuration for which $p_{B}=1.65$ ("upper bound") leads to a mass-loss rate behavior similar to Holzwarth \& Jardine (2007), with $\dot{M} \propto R_{\star}^{2} F_{\mathrm{X}}^{0.5}$ and $\dot{M} \propto \mathrm{Ro}^{-1}$ $\left(p_{\dot{M}}=1\right)$. However, the difference observed in Sect. 3.5 regarding the rotational dependency of $T_{\mathrm{c}}$ and $n_{\mathrm{c}}$ arises partially from a reasonable choice of $\left(\gamma, T_{\odot}\right)$. Indeed, by considering $\gamma=1.22$ and $T_{\odot}=2.93 \mathrm{MK}$, as in their study, we obtain $p_{\mathrm{T}}=0.074$ and $p_{\mathrm{n}}=0.64$, which is close to their published values of $p_{\mathrm{T}}=0.1$ and $p_{\mathrm{n}}=0.6$.

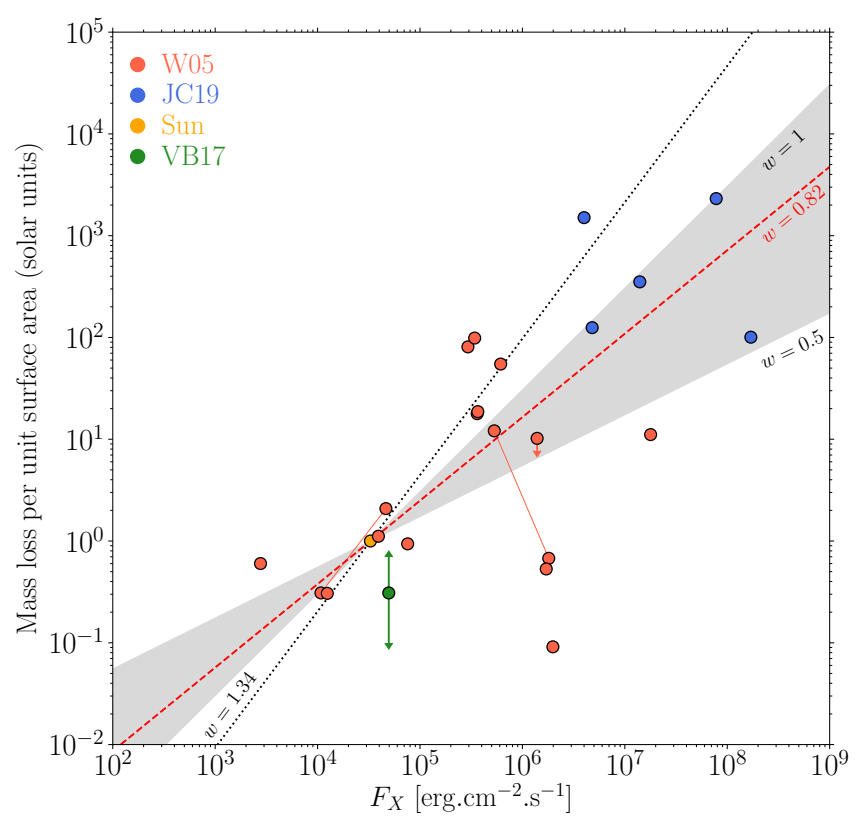

Fig. 6. Mass loss per unit area as a function of the X-ray flux. The red dots come from Wood et al. (2005; W05) and the blue dots are from Jardine \& Collier (2019; JC19). In green: measurements from the atmospheric evaporation of GJ 436b (Vidotto \& Bourrier 2017, VB17). Gray dotted line: Wood et al. (2005) scaling law corresponding to $w=1.34$. Red dashed line: Suzuki et al. (2013) scaling law corresponding to $w=0.82$. The light gray region corresponds to the upper and lower bounds we considered to be consistent with ZDI statistical studies.

Statistical ZDI studies, through the bounds of the $p_{B}$ exponents and prescriptions from spin down considerations, allow us to predict the admissible trends for the $\dot{M}-F_{\mathrm{X}}$ correlation (cf. Eq. (23)). This is shown in Fig. 6 as a light gray area along with the observed $\dot{M} \propto R_{\star}^{2} F_{\mathrm{X}}^{w}$ relationship, which was initially published by Wood et al. (2005). One can notice that the predicted values of the $w$ exponent are compatible with the observations from Wood et al. (2005) and Jardine \& Collier (2019). Furthermore, they present a bias toward active stars with low mass-loss rates, leading to weaker slopes than the Wood et al. (2005) prescription of $w=1.34$ (see the gray dotted line in Fig. 6). Nevertheless, this behavior is in agreement with the results from Suzuki et al. (2013), which predicts an exponent of $w=0.82$ (see the red dashed line in Fig. 6).

\subsubsection{Normalization factors}

If the exponents are fixed thanks to the previous calculations, the normalization of the scaling laws is still an open issue. By default, in the above analytical development, all of the relevant quantities have been normalized to the solar values. However, we need to take additional constraints into account for each physical parameter in a solar configuration.

Regarding the magnetic field, ZDI studies show a significant scatter in the dataset is used to exhibit correlations between the large scale magnetic field and other stellar parameters. Such a dispersion is taken into account here in the magnetic field normalization by considering the See et al. (2017) dataset for which the average dipolar field strength at their value for the solar Rossby number lies between $B_{\odot}=0.6$ and $4 \mathrm{G}$.

The value of $\dot{M}_{\odot}$ is deduced from $B_{\odot}$ by keeping a fixed solar wind torque since $\Gamma_{\text {wind, } \odot} \propto B_{\odot}^{4 m} \dot{M}_{\odot}^{1-2 m}$ (cf. Eq. (11)). This way, 
Table 2. Stellar parameters of $\epsilon$ Eridani.

\begin{tabular}{|c|c|c|}
\hline Star & \multicolumn{2}{|c|}{$\begin{array}{c}\epsilon \text { Eridani } \\
\text { Model inputs }\end{array}$} \\
\hline$M_{\star}\left(M_{\odot}\right)$ & \multicolumn{2}{|c|}{$0.856_{-0.008}^{+0.006} \quad(a)$} \\
\hline$P_{\text {rot }}(d)$ & \multicolumn{2}{|c|}{$11.68^{(a)}$} \\
\hline Age (Gyr) & \multicolumn{2}{|c|}{$0.44^{(a)}$} \\
\hline & Observations & Model outputs \\
\hline$R_{\star}\left(R_{\odot}\right)$ & $0.74 \pm 0.01^{(a)}$ & $0.87^{(b)}$ \\
\hline$L_{\star}\left(L_{\odot}\right)$ & $0.34^{(c)}$ & $0.54^{(b)}$ \\
\hline $\log L_{\mathrm{X}}\left(\mathrm{erg} \mathrm{s}^{-1}\right)$ & $28.32^{(d)}$ & $28.24^{(b)}$ \\
\hline Ro & - & $0.28^{(b)}$ \\
\hline$B_{\star}(\mathrm{G})$ & $6.15-19.8^{(a),(e)}$ & $3-47^{(b)}$ \\
\hline$\dot{M}\left(10^{-14} M_{\odot} \mathrm{yr}^{-1}\right)$ & $30-120^{(d)}$ & $1.9-120^{(b)}$ \\
\hline
\end{tabular}

References. ${ }^{(a)}$ Jeffers et al. (2014). ${ }^{(b)}$ This work (Model). ${ }^{(c)}$ Saumon et al. (1996). ${ }^{(d)}$ Wood et al. (2002). ${ }^{(e)}$ See et al. (2017).

for $m=0.22$, we have $7.9 \times 10^{-15} \leq \dot{M}_{\odot}\left[M_{\odot} \mathrm{yr}^{-1}\right] \leq 1.47 \times$ $10^{-13}$.

The normalization of the coronal temperature and density is determined by performing 1D simulations of a pressure-driven polytropic wind with $\gamma=1.05$, using the starAML routine (Réville et al. 2015b). The value of $T_{\odot}$ is tuned to provide an average solar wind velocity at $1 \mathrm{AU}$ of $444 \mathrm{~km} \mathrm{~s}^{-1}$, leading to $T_{\odot}=1.5 \mathrm{MK}$ (Réville et al. 2016). The density at the base of the solar corona is then computed to be consistent with $T_{\odot}$ and $\dot{M}_{\odot}$, which results in $2.49 \times 10^{7} \leq n_{\odot}\left[\mathrm{cm}^{-3}\right] \leq 4.63 \times 10^{8}$.

Since the exponents of the scaling laws and their normalization factors are well-defined, we can now compare the different prescriptions to observations of individual systems. We focus on ZDI studies that constrain the stellar magnetic field and mass loss measurements from astrospheres' Ly $\alpha$ absorption.

\subsection{A star studied through astrosphere's Ly $\alpha$ absorption and Zeeman-Doppler Imaging: $\epsilon$ Eridani}

We applied our formalism to $\epsilon$ Eridani, a young active $\mathrm{K} 2 \mathrm{~V}$ dwarf, which hosts an exoplanet and a debris disk. We study this individual system by only using the minimal information required in our formalism in order to test the different scaling laws. In practical terms, we only rely on the stellar mass and the rotation period, which are essential when using the different prescriptions (see Table 2 for numerical values of those stellar parameters). An estimate of the age indicates that the star is in the main sequence.

The stellar luminosity, the stellar radius, and the X-ray luminosity can be estimated through Eqs. (12), (13), and (24) (see Table 1 for the associated exponents). The values obtained from those correlations, which are shown in Table 2, are in good agreement with their observed analogs. In practice, those relationships are crucial to estimate $B_{\star}$ and $\dot{M}$ since inaccuracy in the determination of $L_{\mathrm{X}}$ would lead to an erroneous value for the mass-loss rate through the $\dot{M}-F_{\mathrm{X}}$ correlation.

All of those considerations allow us to estimate the stellar magnetic field and the mass loss with our formalism. We illustrate those predictions by the blue bands in Fig. 7. Without further assumptions, our model predicts a broad range of possible $B_{\star}$ (panel A) and $\dot{M}$ (panel B) values. Since we are performing a systematic study of $\epsilon$ Eridani, we first assume that the star follows the observational trends inferred from ZDI studies. Only the exponents situated in the light gray area that are

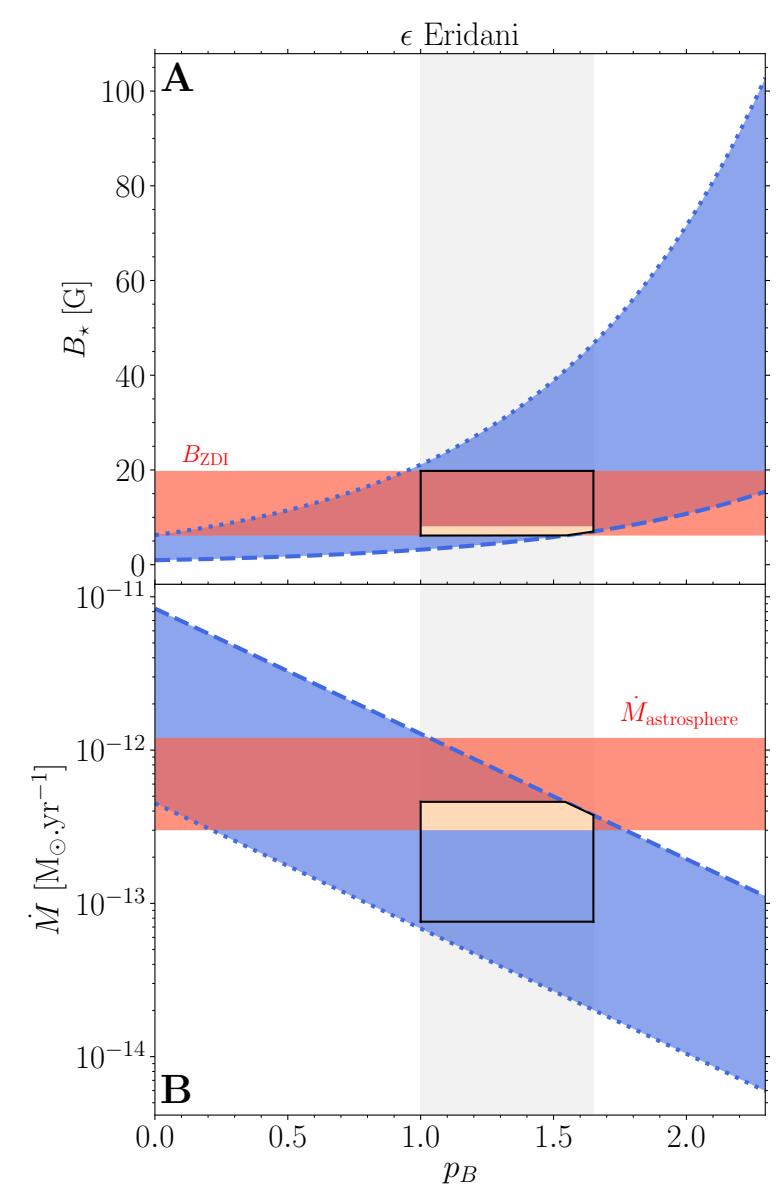

Fig. 7. Panel $A$ : estimate of $B_{\star}$ for $\epsilon$ Eridani as a function of $p_{B}$. Panel $B$ : estimate of $\dot{M}$ as a function of $p_{B}$. In blue: predictions of our prescriptions. Dashed blue lines: normalizations corresponding to $B_{\odot}=0.6 \mathrm{G}$. Dotted blue lines: normalizations corresponding to $B_{\odot}=4$ G. Red bands: observational constraints on the dipolar component of the magnetic field (Jeffers et al. 2014; See et al. 2017) and the massloss rate (Wood et al. 2002). The light gray band corresponds to the upper and lower bounds of $p_{B}$ from a large sample of ZDI studies. The black contours delimit the region of $B_{\star}$ and $\dot{M}$, which is consistent with statistical ZDI studies and individual measurements of the large-scale magnetic field. In beige: estimates consistent with all of the constraints considered.

defined in Fig. 7 are considered $\left(1 \leq p_{B} \leq 1.65\right)$. A modification in the $B_{\star}-$ Ro relationship then corresponds to a horizontal shift in Fig. 7. A change of normalization between $B_{\odot}=0.6 \mathrm{G}$ (dashed blue line in Fig. 7) and $B_{\odot}=4 \mathrm{G}$ (dotted blue line in Fig. 7), which is linked to the scatter in the ZDI studies' dataset, results in a vertical shift in Fig. 7. Browsing this parameter space then gives $3 \leq B_{\star}[\mathrm{G}] \leq 47$ and $1.9 \times 10^{-14} \leq \dot{M}$ $\left[M_{\odot} \mathrm{yr}^{-1}\right] \leq 1.2 \times 10^{-12}$.

The large-scale magnetic field of the star has been monitored for almost seven years, between January 2007 and October 2013, by Jeffers et al. (2014). We only consider the dipolar component of their ZDI maps, leading to field values between 6.15 and $19.8 \mathrm{G}$ (See et al. 2017), thus constraining our estimates of the magnetic field (see the red band at the top of Fig. 7). By taking those observations into account, we can constrain the normalization factors and derive a narrower range for $\dot{M}: 7.59 \times 10^{-14} \leq \dot{M}$ $\left[M_{\odot} \mathrm{yr}^{-1}\right] \leq 4.59 \times 10^{-13}$ (see the black contours in panel $\mathrm{B}$ of Fig. 7).

Furthermore, Wood et al. (2002) measured the mass-loss rate of the star through its astrosphere's Ly $\alpha$ absorption, leading to 
Table 3. Coronal properties of $\epsilon$ Eridani.

\begin{tabular}{lcc}
\hline \hline Scenario & $T_{\mathrm{c}}[\mathrm{MK}]$ & $n_{\mathrm{c}}\left[10^{8} \mathrm{~cm}^{-3}\right]$ \\
\hline Single temperature scaling & 2.83 & $0.34-0.52$ \\
Entropy equilibrium & $1.73-1.85$ & $3.53-3.46$ \\
Single density scaling & $1.58-1.72$ & $6.98-5.62$ \\
\hline
\end{tabular}

$\dot{M} \approx 6 \times 10^{-13} M_{\odot} \mathrm{yr}^{-1}$. Uncertainties in determining this value, for instance regarding interstellar medium properties or wind variability, introduce a systematic error of 0.3 dex in $\log \dot{M}$, that is, a factor of 2 in the mass-loss rate (see Wood et al. 2002 for more details). Therefore, we can assume a mass loss measured through $\epsilon$ Eridani's astrosphere between $3 \times 10^{-13} M_{\odot} \mathrm{yr}^{-1}$ and $1.2 \times 10^{-12} M_{\odot} \mathrm{yr}^{-1}$ (see the red band in panel B of Fig. 7). This constraint further refines the acceptable parameters of our model, which leads to $B_{\star}$ between 6.15 and $8 \mathrm{G}$ (see the beige areas in Fig. 7). It is worth noticing that our $B_{\star}$ and $\dot{M}$ prescriptions are consistent with both statistical approaches and individual measurements, which is evident in the small beige areas. Furthermore, the wind of $\epsilon$ Eridani has been modeled in 3D with an MHD model by Alvarado-Gómez et al. (2016). They find a mass-loss ranging from $2.77 \times 10^{-14}$ to $10.2 \times 10^{-14} M_{\odot} \mathrm{yr}^{-1}$ for January 2010. Our approach is also compatible with these values (see panel B in Fig. 2).

The range of $\dot{M}$ obtained (between $3 \times 10^{-13}$ and $4.59 \times$ $\left.10^{-13} M_{\odot} \mathrm{yr}^{-1}\right)$ allowed us to estimate the coronal temperature $T_{\mathrm{c}}$ and the coronal density $n_{\mathrm{c}}$ for this particular star. Depending on the scenario considered (single density scaling, entropy equilibrium, or single temperature scaling), since the normalization factor $T_{\odot}$ was fixed in Sect. 4.1.3, the range of $p_{B}$ available provides directly upper and lower bounds of $T_{\mathrm{c}}$ with our prescriptions (see Table 1 and Appendix E for numerical values). The range of density is then determined by connecting the lower and upper bounds of $\dot{M}$ and $T_{\mathrm{c}}$. The corresponding numerical values for the coronal properties are shown in Table 3.

One can see that the entropy equilibrium scenario yields slightly higher temperatures compared to the single density scaling hypothesis, therefore resulting in lower densities for a given mass-loss rate. In the single temperature scaling scenario, $T_{\mathrm{c}}$ is independent of the $B_{\star}$ and $\dot{M}$ prescriptions because of the $T_{1}-F_{\mathrm{X}}$ correlation (cf. Eq. (37)). Moreover, here, the star is characterized by a low Rossby number in the unsaturated regime, leading to a higher coronal temperature than those derived from the two other scenarios. Hence, the coronal density, in the case of a single temperature scaling, reaches the lowest values in order to keep a consistent mass loss.

To sum up, we show with $\epsilon$ Eridani that it is possible to have an analytical prediction for the large-scale magnetic field and the mass-loss rate that is in agreement with all of the observational constraints available and, additionally, that it is feasible to infer from the values obtained a range for the coronal properties of the star, according to different scenarios. Furthermore, with a systematic approach, relying on scaling laws and statistical considerations gives a quite large range for $B_{\star}$ and $\dot{M}$, compared to well-constrained quantities coming from individual studies. In the case of $\epsilon$ Eridani, such a guess can deviate from the measured value by a factor of 6 at most in $B_{\star}$ and about one order of magnitude in $\dot{M}$. If for the study of an individual star, additional measurements are required to reduce the interval of confidence, we see how powerful our approach is to guess key trends along with stellar properties in an ensemble approach. Given our minimal set of hypothesis, our formalism provides a good estimate of the relevant quantities from general scaling laws and statistical trends, which is compatible with individual studies.

\section{Conclusions and discussions}

In this paper, we provide power-law prescriptions for all of the relevant parameters required to describe the spin down of solartype stars consistently. We confirm that the magnetic field and the mass loss are involved in a one-to-one correspondence. This is the direct consequence of assuming a generic braking torque parametrization that accounts for both the distribution of stellar rotation periods in open clusters and the Skumanich law in the unsaturated rotation regime. A mass loss-X-ray flux relation coming from astropheres' Ly $\alpha$ absorption (Wood et al. 2005), coupled with the knowledge of the rotational dependency of the $\mathrm{X}$-ray luminosity, allowed us to link the mass-radius dependency of the aforementioned quantities to their rotational dependency. In this way, we show that a magnetic field depending on both the Rossby number and the stellar mass may be required to remain consistent with a whole suite of observational trends. Such an approach allows us to provide upper and lower bounds for the estimates of $B_{\star}$ and $\dot{M}$. The weakest $B_{\star}-$ Ro relationship we considered leads to the following scaling laws:

$$
\begin{aligned}
& B_{\star}[G]=(0.6-4) \times\left(\frac{\mathrm{Ro}}{\mathrm{Ro}_{\odot}}\right)^{-1}\left(\frac{M_{\star}}{M_{\odot}}\right)^{-1.76}, \\
& \dot{M}\left[10^{-14} M_{\odot} \mathrm{yr}^{-1}\right]=(0.79-14.7) \times\left(\frac{\mathrm{Ro}}{\mathrm{Ro}_{\odot}}\right)^{-2}\left(\frac{M_{\star}}{M_{\odot}}\right)^{4} .
\end{aligned}
$$

In the case of the strongest Rossby-dependency of $B_{\star}$ consistent with ZDI studies, we obtain

$$
\begin{aligned}
& B_{\star}[G]=(0.6-4) \times\left(\frac{\mathrm{Ro}}{\mathrm{Ro}_{\odot}}\right)^{-1.65}\left(\frac{M_{\star}}{M_{\odot}}\right)^{-1.04}, \\
& \dot{M}\left[10^{-14} M_{\odot} \mathrm{yr}^{-1}\right]=(0.79-14.7) \times\left(\frac{\mathrm{Ro}}{\mathrm{Ro}_{\odot}}\right)^{-1}\left(\frac{M_{\star}}{M_{\odot}}\right)^{2.9} .
\end{aligned}
$$

Furthermore, given a simple polytropic wind model and an expression of the X-ray luminosity from radiative losses, we were able to go back to the coronal properties by assuming different scenarios linking closed loops to coronal holes. This permits us to consider, in a very simplified way, magnetic geometry effects occurring in stellar atmospheres. Some of these scenarios (namely scenarios 2 and 3, see Sect. 3.5) allow us to reconcile temperature prescriptions deduced from X-ray emission and mass-loss rate constraints, hence providing a fully consistent framework. To demonstrate the usefulness of our study, we applied it to a real star, $\epsilon$ Eridani. We provide estimates of the magnetic field and the mass-loss rate, which are consistent with the different observational constraints, and give a preliminary assessment of its coronal properties. In a saturated rotation regime, we show that a wind torque that depends linearly on the rotation rate implies a magnetic field and a mass-loss rate independent of the Rossby number. We then find a similar behavior for the coronal temperature and density, depending on the physical scenario we adopted to connect open and closed regions.

We managed to infer all of the exponents of our scaling laws from the $B_{\star}-$ Ro relation. Furthermore, in this paper we adopted an observational point of view to constrain the $p_{B}$ exponent $\left(B_{\star} \propto \mathrm{Ro}^{-p_{B}}\right)$ by relying on the large sample of ZDI studies. One can also use theoretical dynamo scalings to determine the rotational dependency of the magnetic field (Augustson et al. 2017). However, we have to bear in mind that 
those prescriptions are based on the magnetic energy content in the stellar interior over a wide spectral range, whereas in this work we consider the large scale magnetic field at the stellar surface. To link the two approaches, one can fall back on a filling factor, which may depend on stellar rotation (See et al. 2019).

Stellar metallicity was not directly taken into account in our study. However, it could significantly affect the coronal density and the stellar mass loss (Suzuki 2018), thus influencing the wind braking torque. In our work, the effect of metallicity on the stellar structure is included in the Rossby dependency. Therefore, studying the influence of metallicity on the rotational evolution of solar-type stars may be a promising avenue to test different torque prescriptions.

Furthermore, coronal temperature and density are directly linked to the choice of a wind model. In this work, to connect the mass-loss rate to the coronal properties, we relied on an expression for $\dot{M}$ valid in the case of a nonmagnetized outflow of a pressure-driven polytropic wind. Assuming a more realistic model, such as a magnetized wind, would introduce corrections in the mass loss expression due to the magnetocentrifugal effect (Preusse et al. 2005; Johnstone 2017) involving, for instance, the stellar rotation rate and the Alfvén radius. This may lead to implicit relations between the different prescriptions. Given that our formalism relies on the Matt et al. (2015) wind braking torque, which does not take such an effect into account, a pressure-driven hydrodynamic polytropic wind may be more suitable in this context to keep a consistent model. Modifications to the wind torque and the mass-loss rate may be required to deal with very fast rotators. More complex models could also be investigated, such as a polytropic gas with a spatially varying polytropic index (Johnstone et al. 2015b).

In this paper, we do not consider a slow and a fast wind, which would be a way of improving our model ever more. Indeed, for a pressure-driven wind, higher temperatures lead to faster winds. However, an anticorrelation is observed between the terminal speed of the two components of the solar wind and the coronal temperature of the source region, which may be due to a difference in the altitude of the heating region between the fast and the slow component (Geiss et al. 1995; Schwadron \& McComas 2003). Therefore, more realistic wind acceleration processes, including the influence of coronal heating, have to be taken into account to deal with a fast and slow wind (Réville et al. 2020; Riley et al. 2019).

We show that the $p_{\mathrm{L}}$ exponent $\left(L_{\mathrm{X}} \propto L_{\star} \mathrm{Ro}^{-p_{\mathrm{L}}}\right)$ is one of the most important parameters allowing us to constrain all of the different prescriptions efficiently, especially the $B_{\star}$ and $\dot{M}$ massradius dependency as well as the expression of the coronal properties. This way, uncertainty as to this exponent (Pizzolato et al. 2003; Wright et al. 2011) may lead to a significant scatter in our scaling laws. Therefore, our prescriptions could be significantly tightened if the interval of confidence of the $p_{\mathrm{L}}$ exponent could be reduced.

In the case of evolved stars, the decrease of the wind braking efficiency (van Saders et al. 2016) was not studied in this work. However, the influence of this phenomenon on the different wind parameters and its eventual inconsistency with other observational constraints may be an application of our formalism. It could be possible to introduce a "resaturation" regime at high Rossby numbers (Sadeghi Ardestani et al. 2017) and to look for hints of breaking of gyrochonology in the physical quantities involved in stellar spin down.

Acknowledgements. We would like to thank the anonymous referee and Sean Matt for helpful comments and suggestions regarding our work. The authors acknowledge funding from the European Union's Horizon-2020 research and innovation programme (Grant Agreement no. 776403 ExoplANETS-A). A.S and A.S.B. acknowledge funding by ERC WHOLESUN 810218 grant, INSU/ PNST, CNES-PLATO and CNES Solar Orbiter. A.S. acknowledges funding from the Programme National de Planétologie (PNP). This work benefited from discussions within the international team "The Solar and Stellar Wind Connection: Heating processes and angular momentum loss", supported by the International Space Science Institute (ISSI). We also thank Victor Réville, Manuel Güdel, Colin Johnstone, Aurélie Astoul and Kyle Augustson for useful discussions.

\section{References}

Agüeros, M. A., Covey, K. R., Lemonias, J. J., et al. 2011, ApJ, 740, 110 Alvarado-Gómez, J. D., Hussain, G. A. J., Cohen, O., et al. 2016, A\&A, 594 A95

Amard, L., Palacios, A., Charbonnel, C., et al. 2019, A\&A, 631, A77

Armitage, P. J., \& Clarke, C. J. 1996, MNRAS, 280, 458

Aschwanden, M. J. 2004, Physics of the Solar Corona. An Introduction (Chichester, UK: Praxis Publishing Ltd)

Augustson, K., Mathis, S., \& Brun, A. S. 2017, ArXiv e-prints [arXiv:1701.02582]

Barnes, S. A. 2003, ApJ, 586, 464

Barnes, S., \& Sofia, S. 1996, ApJ, 462, 746

Benbakoura, M., Réville, V., Brun, A. S., Le Poncin-Lafitte, C., \& Mathis, S. 2019, A\&A, 621, A124

Blackman, E. G., \& Owen, J. E. 2016, MNRAS, 458, 1548

Bouvier, J., Forestini, M., \& Allain, S. 1997, A\&A, 326, 1023

Brandenburg, A., \& Saar, S. H. 2000, in Stellar Clusters and Associations: Convection, Rotation, and Dynamos, eds. R. Pallavicini, G. Micela, \& S. Sciortino (San Francisco, CA: ASP), ASP Conf. Ser., 198, 381

Brun, A. S., Miesch, M. S., \& Toomre, J. 2004, ApJ, 614, 1073

Brun, A. S., Browning, M. K., Dikpati, M., Hotta, H., \& Strugarek, A. 2015, Space Sci. Rev., 196, 101

Brun, A. S., Strugarek, A., Varela, J., et al. 2017, ApJ, 836, 192

Cranmer, S. R., \& Saar, S. H. 2011, ApJ, 741, 54

Cranmer, S. R., Gibson, S. E., \& Riley, P. 2017, Space Sci. Rev., 212, 1345

Durney, B. R., \& Latour, J. 1978, Geophys. Astrophys. Fluid Dyn., 9, 241

Finley, A. J., \& Matt, S. P. 2017, ApJ, 845, 46

Finley, A. J., \& Matt, S. P. 2018, ApJ, 854, 78

Gallet, F., \& Bouvier, J. 2015, A\&A, 577, A98

Garraffo, C., Drake, J. J., \& Cohen, O. 2016, A\&A, 595, A110

Garraffo, C., Drake, J. J., Dotte, A., et al. 2018, ApJ, 862, 90

Geiss, J., Gloeckler, G., \& von Steiger, R. 1995, Space Sci. Rev., 72, 49

Güdel, M. 2007, Liv. Rev. Sol. Phys., 4, 3

Heyvaerts, J., \& Priest, E. R. 1983, A\&A, 117, 220

Holzwarth, V., \& Jardine, M. 2007, A\&A, 463, 11

Ivanova, N., \& Taam, R. E. 2003, ApJ, 599, 516

Jardine, M., \& Collier, Cameron A. 2019, MNRAS, 482, 2853

Jeffers, S. V., Petit, P., Marsden, S. C., et al. 2014, A\&A, 569, A79

Johns-Krull, C. M., \& Valenti, J. A. 2000, in Measurements of Stellar Magnetic Fields. Stellar Clusters and Associations: Convection, Rotation, and Dynamos, eds. R. Pallavicini, G. Micela, \& S. Sciortino (San Francisco: Astronomical Society of the Pacific), ASP Conf. Ser., 198, 371

Johnstone, C. P. 2017, A\&A, 598, A24

Johnstone, C. P., \& Güdel, M. 2015, A\&A, 578, A129

Johnstone, C. P., Güdel, M., Brott, I., \& Lüftinger, T. 2015a, A\&A, 577, A28

Johnstone, C. P., Güdel, M., Lüftinger, T., Toth, G., \& Brott, I. 2015b, A\&A, 577, A27

Kawaler, S. D. 1988, ApJ, 333, 236

Kippenhahn, R., \& Weigert, A. 1994, Stellar Structure and Evolution (Berlin: Springer)

Krishnamurthi, A., Pinsonneault, M. H., Barnes, S., \& Sofia, S. 1997, ApJ, 480, 303

Lamers, H. J. G. L. M., \& Cassinelli, J. P. 1999, Introduction to Stellar Winds (Cambridge, UK: Cambridge University Press)

Landin, N. R., Mendes, L. T. S., \& Vaz, L. P. R. 2010, A\&A, 510, A46

Lorenzo-Oliveira, D., Meléndez, J., Galarza, J. Y., et al. 2019, MNRAS, 485, L68

Mathioudakis, M., Jess, D. B., \& Erdélyi, R. 2013, Space Sci. Rev., 175, 1

McQuillan, A., Mazeh, T., \& Aigrain, S. 2014, ApJS, 211, 24

Matt, S., \& Pudritz, R. E. 2008, ApJ, 678, 1109

Matt, S. P., Pinzón, G., Greene, T. P., \& Pudritz, R. E. 2012, ApJ, 745, 101

Matt, S. P., Brun, A.-S., Baraffe, I., Bouvier, J., \& Chabrier, G. 2015, ApJ, 799, L23

Mestel, L. 1968, MNRAS, 138, 359

Mestel, L., \& Spruit, H. C. 1987, MNRAS, 226, 57

Montesinos, B., \& Jordan, C. 1993, MNRAS, 264, 900 
Morel, P., \& Lebreton, Y. 2008, Ap\&SS, 316, 61

Noyes, R. W., Weiss, N. O., \& Vaughan, A. H. 1984, ApJ, 287, 769

Ó Fionnagain, D., \& Vidotto, A. A. 2018, MNRAS, 476, 2465

Parker, E. N. 1958, ApJ, 128, 664

Petit, P., Dintrans, B., Aurière, M., et al. 2008, in SF2A-2014: SF2A2008: Proceedings of the Annual meeting of the French Society of Astronomy and Astrophysics, eds. C. Charbonnel, F. Combes, \& R. Samadi, 523

Pevtsov, A. A., Fisher, G. H., Acton, L. W., et al. 2003, ApJ, 598, 1387

Pizzolato, N., Maggio, A., Micela, G., Sciortino, S., \& Ventura, P. 2003, A\&A, 397, 147

Preibisch, T. 1997, A\&A, 320, 525

Preusse, S., Kopp, A., Büchner, J., \& Motschmann, U. 2005, A\&A, 434, 1191

Reiners, A. 2012, Liv. Rev. Sol. Phys., 9, 1

Reiners, A., \& Mohanty, S. 2012, ApJ, 746, 43

Reiners, A., Schüssler, M., \& Passegger, V. M. 2014, ApJ, 794, 144

Réville, V., Brun, A.-S., Matt, S. P., Strugarek, A., \& Pinto, R. F. 2015a, ApJ, 798, 116

Réville, V., Brun, A.-S., Strugarek, A., et al. 2015b, ApJ, 814, 99

Réville, V., Folsom, C. P., Strugarek, A., \& Brun, A. S. 2016, ApJ, 832, 145

Réville, V., Tenerani, A., \& Velli, M. 2018, ApJ, 866, 38

Réville, V., Velli, M., Panasenco, O., et al. 2020, ApJS, 246, 24

Riley, P., Linker, J. A., Mikic, Z., et al. 2019, ApJ, 884, 18

Rosner, R., Tucker, W. H., \& Vaiana, G. S. 1978, ApJ, 220, 643

Sadeghi Ardestani, L., Guillot, T., \& Morel, P. 2017, MNRAS, 472, 2590

Sakurai, T. 1985, A\&A, 152, 121
Saumon, D., Hubbard, W. B., Burrows, A., et al. 1996, ApJ, 460, 993

Schatzman, E. 1962, Ann. Astrophys., 25, 18

Schwadron, N. A., \& McComas, D. J. 2003, ApJ, 599, 1395

See, V., Jardine, M., Vidotto, A. A., et al. 2014, A\&A, 570, A99

See, V., Jardine, M., Vidotto, A. A., et al. 2017, MNRAS, 466, 1542

See, V., Matt, S. P., Folsom, C. P., et al. 2019, ApJ, 876, 118

Skumanich, A. 1972, ApJ, 171, 565

Skumanich, A. 2019, ApJ, 878, 35

Suzuki, T. K. 2018, PASJ, 70, 34

Suzuki, T. K., \& Inutsuka, S. 2006, J. Geophys. Res., 111, A06101

Suzuki, T. K., Imada, S., Kataoka, R., et al. 2013, PASJ, 65, 98

Tu, L., Johnstone, C. P., Güdel, M., \& Lammer, H. 2015, A\&A, 577, L3

Velli, M., Grappin, R., \& Mangeney, A. 1989, Phys. Rev. Lett., 63, 1807

Vidotto, A. A., \& Bourrier, V. 2017, MNRAS, 470, 4026

Vidotto, A. A., Gregory, S. G., Jardine, M., et al. 2014, MNRAS, 441, 2361

Weber, E. J., \& Davis, Jr., L. 1967, ApJ, 148, 217

Wedemeyer-Böhm, S., Lagg, A., \& Nordlund, A. 2009, Space Sci. Rev., 144, 317

Wright, N. J., Drake, J. J., Mamajek, E. E., \& Henry, G. W. 2011, ApJ, 743, 48

van Saders, J. L., Ceillier, T., Metcalfe, T. S., et al. 2016, Nature, 529, 181

Wood, B. E., Müller, H.-R., Zank, G. P., \& Linsky, J. L. 2002, ApJ, 574, 412

Wood, B. E., Müller, H.-R., Zank, G. P., Linsky, J. L., \& Redfield, S. 2005, ApJ, $628, \mathrm{~L} 143$

Wood, B. E., Laming, J. M., Warren, H. P., \& Poppenhaeger, K. 2018, ApJ, 862, 66

Zhang, M., \& Penev, K. 2014, ApJ, 787, 131 


\section{Appendix A: Mass loss for a Parker polytropic wind}

The goal of this section is to compute the stellar mass loss by assuming a radial polytropic pressure-driven outflow, with an index $\gamma$. We follow Lamers \& Cassinelli (1999) in the remainder of this section. The wind accelerates with distance and its velocity $v$ reaches the speed of sound $c_{\mathrm{s}}$ at a critical radius $r_{\mathrm{c}}=G M_{\star} / 2 c_{\mathrm{s}}^{2}\left(r_{\mathrm{c}}\right)$ (Parker 1958). The momentum equation leads to the following integral formulation:

$e_{\gamma}=\frac{v^{2}}{2}+\frac{c_{\mathrm{s}}^{2}}{\gamma-1}-\frac{G M_{\star}}{r}=C^{\mathrm{te}}$.

Such a constant can be estimated at the critical radius as follows:

$e_{\gamma}=\frac{5-3 \gamma}{\gamma-1} \frac{G M_{\star}}{4 r_{\mathrm{c}}}$.

Furthermore, from the mass conservation, we know that $\rho v r^{2}$, where $\rho$ is the density of the wind, is a constant. As $c_{\mathrm{s}}^{2} \propto \rho^{\gamma-1}$ for a polytrope, the speed of sound adheres to the following expression:

$\frac{c_{\mathrm{s}}^{2}}{c_{\mathrm{s}}^{2}\left(r_{\mathrm{c}}\right)} \propto\left(\frac{v}{c_{\mathrm{s}}\left(r_{\mathrm{c}}\right)}\right)^{1-\gamma}\left(\frac{r}{r_{\mathrm{c}}}\right)^{2-2 \gamma}$.

By defining $w=v / c_{\mathrm{s}}\left(r_{\mathrm{c}}\right)$ and $x=r / r_{\mathrm{c}}$, Eq. (A.1) becomes:

$w^{\gamma+1}-w^{\gamma-1}\left(\frac{4}{x}+\frac{5-3 \gamma}{\gamma-1}\right)+\frac{2}{\gamma-1} x^{2-2 \gamma}=0$.

By definition of the critical radius, we have:

$c_{\mathrm{s}}^{2}\left(r_{\mathrm{c}}\right)=\frac{G M_{\star}}{2 r_{\mathrm{c}}}=\Lambda x_{0} c_{\mathrm{s}}^{2}\left(R_{\star}\right)$,

where $x_{0}=R_{\star} / r_{\mathrm{c}}$ and $\Lambda=v_{\mathrm{esc}}^{2} / 4 c_{\mathrm{s}}^{2}\left(R_{\star}\right)$. Therefore, thanks to Eq. (A.3), assessing Eq. (A.4) at the base of the corona, which is assumed to be situated approximately at the stellar radius, gives the following relationship:

$\left(\Lambda x_{0}^{3-2 \gamma}\right)^{\frac{\gamma+1}{\gamma-1}}-\left(\Lambda x_{0}^{3-2 \gamma}\right)\left(\frac{4}{x_{0}}+\frac{5-3 \gamma}{\gamma-1}\right)+\frac{2}{\gamma-1} x_{0}^{2-2 \gamma}=0$.

If we assume that $c_{\mathrm{s}}^{2}\left(R_{\star}\right) \ll v_{\mathrm{esc}}^{2}$, that is, $x_{0} \ll 1$ and $\Lambda \gg 1$, we find the following by neglecting the first term in Eq. (A.6) :

$x_{0}=\frac{2-4(\gamma-1) \Lambda}{(5-3 \gamma) \Lambda}$.

At the base of the corona, we can assume that $w \ll 1$. Therefore, by neglecting the first term, Eq. (A.4) becomes:

$w\left(R_{\star}\right)=\left[\frac{2}{\gamma-1} x_{0}^{3-2 \gamma}\left(4+\frac{5-3 \gamma}{\gamma-1} x_{0}\right)^{-1}\right]^{\frac{1}{\gamma-1}}=\Lambda^{\frac{1}{\gamma-1}} x_{0}^{\frac{3-2 \gamma}{\gamma-1}}$.

Then the wind speed at the same distance can be expressed as:

$v\left(R_{\star}\right)=c_{\mathrm{S}}\left(r_{\mathrm{c}}\right) w\left(R_{\star}\right)=c_{\mathrm{s}}\left(R_{\star}\right) w\left(R_{\star}\right)^{\frac{\gamma+1}{2}} x_{0}^{\gamma-1}$.

It is now possible to estimate the mass loss as follows:

$$
\begin{aligned}
\dot{M} & =4 \pi \rho\left(R_{\star}\right) R_{\star}^{2} \nu\left(R_{\star}\right) \\
& =2 \pi m_{\mathrm{p}} n_{\mathrm{c}} R_{\star}^{2} c_{\mathrm{s}}\left(R_{\star}\right) \Lambda^{\frac{\gamma+1}{2(\gamma-1)}} x_{0}^{\frac{5-3 \gamma}{2(\gamma-1)}} .
\end{aligned}
$$

As $c_{\mathrm{s}}\left(R_{\star}\right)=\sqrt{2 \gamma k_{\mathrm{B}} T_{\mathrm{c}} / m_{\mathrm{p}}}$ for a fully ionized wind, the mass loss has the following dependencies:

$\dot{M} \propto\left(\frac{M_{\star}}{M_{\odot}}\right)^{2}\left(\frac{n_{\mathrm{c}}}{n_{\odot}}\right)\left(\frac{T_{\mathrm{c}}}{T_{\odot}}\right)^{-\frac{3}{2}}\left[1-\frac{T_{\min , \odot}}{T_{\odot}} \frac{M_{\star}}{M_{\odot}} \frac{R_{\odot}}{R_{\star}} \frac{T_{\odot}}{T_{\mathrm{c}}}\right]^{\frac{5-3 \gamma}{2(\gamma-1)}}$,

with $T_{\text {min, } \odot}=(1-1 / \gamma) G m_{\mathrm{p}} M_{\odot} / 2 k_{\mathrm{B}} R_{\odot} \approx 11 \times 10^{6}(1-1 / \gamma) \mathrm{K}$.

\section{Appendix B: X-ray luminosity from radiative losses}

The goal of this section is to compute the X-ray luminosity of the star from coronal properties. If we assume that the corona is fully ionized and optically thin, the X-ray luminosity emitted by a volume of electrons through free-free radiation can be expressed as (Aschwanden 2004; See et al. 2014):

$L_{\mathrm{X}}=\Lambda\left(\bar{T}_{1}\right) \int_{\text {deadzone }} n_{1}^{2} \mathrm{~d} V \propto \Lambda\left(\bar{T}_{1}\right) \bar{n}_{1}^{2} R_{\star}^{3}\left(\frac{r_{\mathrm{dz}}^{3}}{R_{\star}^{3}}-1\right)$,

where $\bar{T}_{1}$ and $\bar{n}_{1}$ are the mean temperature and the mean density in the dead zone, respectively, and $r_{\mathrm{dz}}$ is the radius of the dead zone and $\Lambda\left(\bar{T}_{1}\right)$ is the radiative loss function (Rosner et al. 1978; Aschwanden 2004; Blackman \& Owen 2016), estimated as:

$\Lambda\left(\bar{T}_{1}\right)\left[\mathrm{erg} \mathrm{cm} \mathrm{s}^{-1}\right]=10^{-17.73} \bar{T}_{1}^{-\frac{2}{3}}$.

This prescription is assumed to be accurate for $\bar{T}_{1} \approx 2-10 \mathrm{MK}$ and acceptable as an average down to $0.4 \mathrm{MK}$.

To estimate the radius of the dead zone, we assume a dipolar magnetic field in the closed-field region, where the pressure gradient and the centrifugal force are not strong enough to distort the field lines. The closed loops then trap hot gas and prevent the flow of a stellar wind, allowing us to neglect the ram pressure of the gas in this area. Here, the edge of the dead zone is assimilated to a limit of confinement of the plasma and therefore can be estimated with the following pressure equilibrium:

$p_{\mathrm{th}}\left(r_{\mathrm{dz}}\right)=p_{\text {mag }}\left(r_{\mathrm{dz}}\right)$,

where $p_{\mathrm{th}}\left(r_{\mathrm{dz}}\right), p_{\mathrm{th}}\left(r_{\mathrm{dz}}\right)$ are the thermal and the magnetic pressures of the gas that are estimated at the edge of the dead zone, respectively. Since we consider a dipolar magnetic field, Eq. (B.3) becomes:

$p_{\text {th }}\left(r_{\mathrm{dz}}\right)=\frac{B_{\star}^{2}}{2 \mu_{0}}\left(\frac{R_{\star}}{r_{\mathrm{dz}}}\right)^{6}$.

If we consider that the dead zone is filled with an ideal gas of constant temperature $\bar{T}_{1}=T_{1}$ and a density at the edge of the dead zone that evolves similarly to the density at the base of the corona $n_{1}$, that is, $n_{1}\left(r_{\mathrm{dz}}\right)=K n_{1}$ in which $K$ is a constant independent of any stellar parameter, then $p_{\mathrm{th}}\left(r_{\mathrm{dz}}\right)=n_{\mathrm{l}}\left(r_{\mathrm{dz}}\right) k_{\mathrm{B}} T_{1}=$ $K n_{1} k_{\mathrm{B}} T_{1}$. The radius of the dead zone then becomes:

$\frac{r_{\mathrm{dz}}}{R_{\star}}=\frac{r_{\mathrm{dz}, \odot}}{R_{\odot}}\left(\frac{B_{\star}}{B_{\odot}}\right)^{\frac{1}{3}}\left(\frac{n_{1}}{n_{1, \odot}}\right)^{-\frac{1}{6}}\left(\frac{T_{1}}{T_{1, \odot}}\right)^{-\frac{1}{6}}$,

where:

$\frac{r_{\mathrm{dz}, \odot}}{R_{\odot}}=\left(\frac{1}{K} \frac{B_{\odot}^{2}}{2 \mu_{0}} \frac{1}{k_{\mathrm{B}} n_{1, \odot} T_{1, \odot}}\right)^{\frac{1}{6}}$.

If we assume that $\bar{n}_{1}$ varies the same way as the density at the base of the corona, we can express the X-ray luminosity as a function of more explicit stellar parameters as follows:

$L_{\mathrm{X}} \propto\left(\frac{T_{\odot}}{T_{1}}\right)^{\frac{2}{3}}\left(\frac{n_{1}}{n_{1, \odot}}\right)^{2}\left(\frac{R_{\star}}{R_{\odot}}\right)^{3}\left[\left(\frac{r_{d z, \odot}}{R_{\odot}}\right)^{3}\left(\frac{B_{\star}}{B_{\odot}}\right)\left(\frac{n_{1, \odot}}{n_{1}}\right)^{\frac{1}{2}}\left(\frac{T_{1, \odot}}{T_{1}}\right)^{\frac{1}{2}}-1\right]$.

By assuming $r_{\mathrm{dz}}^{3} / R_{\star}^{3} \gg 1$, the $\mathrm{X}$-ray luminosity becomes:

$L_{\mathrm{X}} \propto\left(\frac{R_{\star}}{R_{\odot}}\right)^{3}\left(\frac{B_{\star}}{B_{\odot}}\right)\left(\frac{n_{1}}{n_{1, \odot}}\right)^{\frac{3}{2}}\left(\frac{T_{1}}{T_{1, \odot}}\right)^{-\frac{7}{6}}$. 


\section{Appendix C: Conditions on the coronal temperature to generate a transsonic wind}

The goal of this section is to investigate the consequences of the positiveness of the mass-loss rate on the coronal temperature. In coronal holes, we assume a correlation between the X-ray flux and the coronal temperature $T_{\mathrm{c}}$, which is similar to the closed loops case

$T_{\mathrm{c}} \propto F_{\mathrm{X}}^{j}$,

where the exponent $j$, which is different from $j_{1}$ because of the possible difference of heating efficiency between open-field and closed-field regions, has to be determined. As for $T_{1}$, we can express $T_{\mathrm{c}}$ as a function of the Rossby number and the stellar mass

$T_{\mathrm{c}} \propto \mathrm{Ro}^{-p_{\mathrm{L}} j} M_{\star}^{(\eta-2 \xi) j}$,

which leads to constraints similar to those obtained in Sect. 3.2, namely

$p_{\mathrm{T}}=p_{\mathrm{L}} j$,

$\xi r_{\mathrm{T}}+m_{\mathrm{T}}=(\eta-2 \xi) j$.

By eliminating the $j$ exponent, the combinated mass-radius dependency of $T_{\mathrm{c}}$ can be inferred from its Rossby dependency with

$\xi r_{\mathrm{T}}+m_{\mathrm{T}}=\frac{\eta-2 \xi}{p_{\mathrm{L}}} p_{\mathrm{T}}$.

In the case of a pressure-driven radial polytropic wind, the coronal temperature has to be greater than a threshold value to keep a positive mass-loss rate, according to Eq. (10):

$\frac{T_{\mathrm{c}}}{T_{\odot}} \frac{R_{\star}}{R_{\odot}} \frac{M_{\odot}}{M_{\star}}>\frac{T_{\mathrm{min}, \odot}}{T_{\odot}}$,

which with Eq. (41) gives

$\left(\frac{\mathrm{Ro}}{\mathrm{Ro}_{\odot}}\right)^{-p_{\mathrm{T}}}\left(\frac{M_{\star}}{M_{\odot}}\right)^{\xi\left(r_{\mathrm{T}}+1\right)+m_{\mathrm{T}}-1}>\frac{T_{\min , \odot}}{T_{\odot}}$.

Here, we consider stars with $0.5 M_{\odot} \leq M_{\star} \leq 1.4 M_{\odot}$, which correspond to F, G, and $\mathrm{K}$ spectral types, and a Rossby number that is less than $10 \mathrm{Ro}_{\odot}$. In order to keep a well-defined mass-loss rate for all of those stars, it is necessary to fulfill the following condition:

$10^{-p_{\mathrm{T}}} m_{\lim }^{\xi\left(r_{\mathrm{T}}+1\right)+m_{\mathrm{T}}-1}>\frac{T_{\mathrm{min}, \odot}}{T_{\odot}}$,

where $m_{\lim }$ is equal to 0.5 if $\xi\left(r_{\mathrm{T}}+1\right)+m_{\mathrm{T}}-1>0$ and 1.4 otherwise. This defines a maximal value for the $p_{\mathrm{T}}$ exponent, considering the range of stellar masses and Rossby numbers considered:

$p_{\mathrm{T}}<\frac{(\xi-1) \log _{10}\left(m_{\mathrm{lim}}\right)-\log _{10}\left(\frac{T_{\min , \odot}}{T_{\odot}}\right)}{1-\frac{\eta-2 \xi}{p_{\mathrm{L}}} \log _{10}\left(m_{\mathrm{lim}}\right)} \equiv p_{\mathrm{T}, \max }$.

This maximal $p_{\mathrm{T}}$ is shown in Fig. C.1. One can notice that $p_{\mathrm{T} \text {,max }}$ decreases with the adiabatic index $\gamma$ because the minimal temperature required to generate a transsonic wind increases with $\gamma$. The $p_{\mathrm{T}, \max }$ exponent then vanishes at a certain value of $\gamma$, beyond which it is not possible to obtain a consistent power-law prescription of $T_{\mathrm{c}}$ without changing the value of $T_{\odot}$.

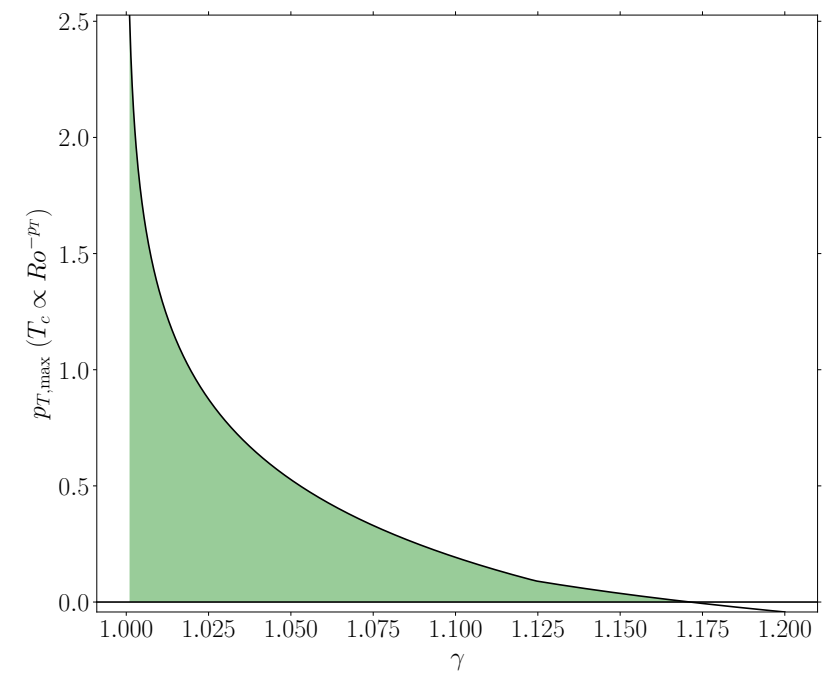

Fig. C.1. Maximal value of $p_{\mathrm{T}}$ needed to keep a positive mass-loss rate, as a function of the adiabatic index $\gamma$, for $\eta=4, \xi=0.9, p_{\mathrm{L}}=2, T_{\odot}=$ 1.5 MK. In green: consistent values of $p_{\mathrm{T}}$.

\section{Appendix D: Power-law expression of the coronal density from wind model considerations}

For a pressure-driven polytropic wind, the coronal density $n_{\mathrm{c}}$ can be inferred from the mass-loss rate as

$n_{\mathrm{c}} \propto \mathrm{Ro}^{-p_{\dot{M}}} R_{\star}^{r_{\dot{M}}} M_{\star}^{m_{\dot{M}}-2} T_{\mathrm{c}}^{\frac{3}{2}}\left[1-\frac{T_{\min , \odot}}{T_{\odot}} \frac{M_{\star}}{M_{\odot}} \frac{R_{\odot}}{R_{\star}} \frac{T_{\odot}}{T_{\mathrm{c}}}\right]^{\frac{3 \gamma-5}{2(\gamma-1)}}$.

In order to simplify this expression, we define $X=\frac{M_{\star}}{M_{\odot}} \frac{R_{\odot}}{R_{\star}} \frac{T_{\odot}}{T_{\mathrm{c}}}$ and

$Q(X)=\left[1-\frac{T_{\min , \odot}}{T_{\odot}} X\right]^{\frac{3 \gamma-5}{2(\gamma-1)}}$.

To approximate this function by a power law, which can be motivated by the $n_{1}$ prescription for instance, we introduce the quantity

$F(\gamma, X)=\frac{\partial[\ln Q(X)]}{\partial[\ln X]}$.

The expression of the function $Q$ then gives

$F(\gamma, X)=\frac{3 \gamma-5}{2(\gamma-1)} \frac{\partial\left[\ln \left(1-\frac{T_{\min , \odot}}{T_{\odot}} X\right)\right]}{\partial[\ln X]}$.

During the main sequence, if we assume a stellar radius that is approximately proportional to the mass of the star, that is, $\xi \approx 1$, we can consider that $M_{\star} R_{\odot} / M_{\odot} R_{\star} \approx 1$. Furthermore, for small values of $p_{\mathrm{T}}$ (and small values of $\xi r_{\mathrm{T}}+m_{\mathrm{T}}$ through the $T_{\mathrm{c}}-F_{\mathrm{X}}$ correlation), which is required to generate a transsonic polytropic wind, we can assume that the coronal temperature only presents small variations for the stellar parameters we consider, leading to $T_{\mathrm{c}} / T_{\odot} \approx 1$ in $F(\gamma, X)$. This way, $X \approx 1$ and

$F(\gamma, X) \approx \frac{5-3 \gamma}{2(\gamma-1)} \frac{T_{\min , \odot} / T_{\odot}}{1-T_{\min , \odot} / T_{\odot}} \equiv F(\gamma)$.

We can therefore express the coronal density as

$n_{\mathrm{c}} \propto\left(\frac{\mathrm{Ro}}{\mathrm{Ro}_{\odot}}\right)^{-p_{\mathrm{n}}}\left(\frac{R_{\star}}{R_{\odot}}\right)^{r_{\mathrm{n}}}\left(\frac{M_{\star}}{M_{\odot}}\right)^{m_{\mathrm{n}}}$, 
with

$p_{\mathrm{n}}=p_{\dot{M}}+\left(\frac{3}{2}-F(\gamma)\right) p_{\mathrm{T}}$,

$r_{\mathrm{n}}=r_{\dot{M}}+\frac{3}{2} r_{\mathrm{T}}-F(\gamma)\left(1+r_{\mathrm{T}}\right)$,

$m_{\mathrm{n}}=m_{\dot{M}}-2+\frac{3}{2} m_{\mathrm{T}}-F(\gamma)\left(m_{\mathrm{T}}-1\right)$

\section{Appendix E: Exponents for other scenarios}

Table E.1. Parameters defining the $\Gamma_{\text {wind }}, B_{\star}, \dot{M}, T_{\mathrm{c}}$, and $n_{\mathrm{c}}$ prescriptions for $p_{B}=1$ and $p_{B}=1.65$ in the single temperature scaling sce-

(D.9)

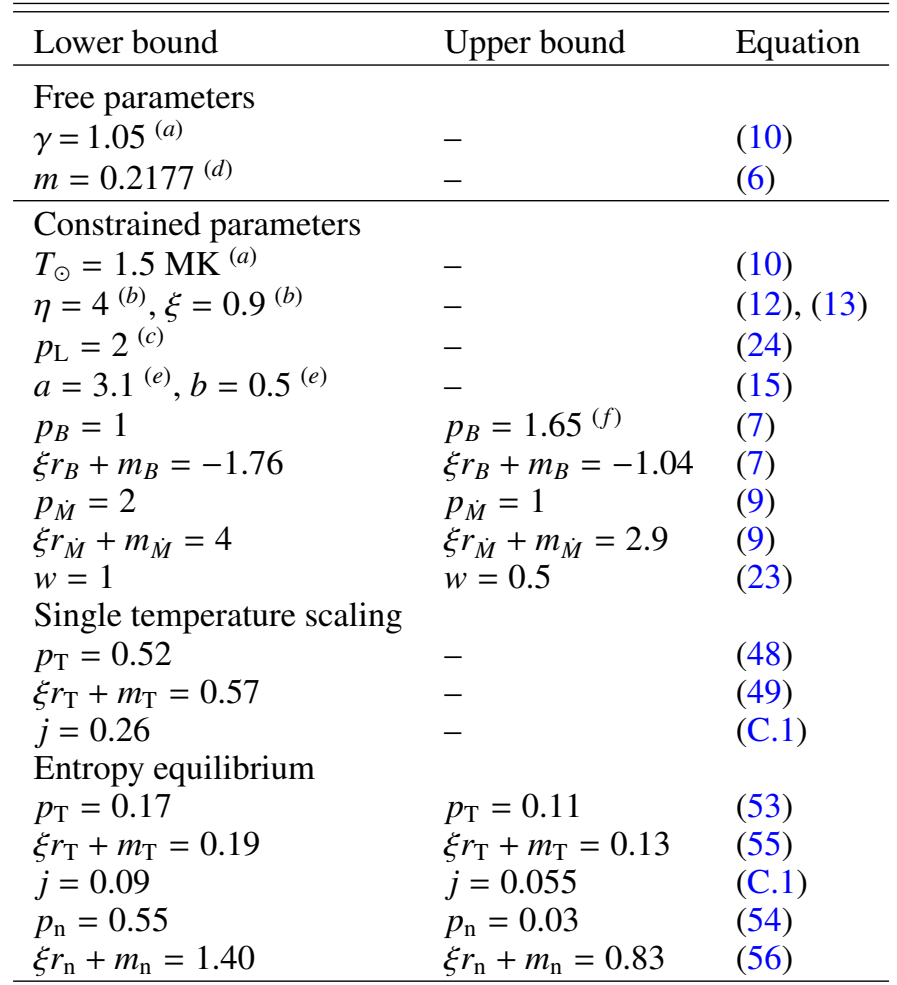

References. ${ }^{(a)}$ Réville et al. (2015b). ${ }^{(b)}$ Kippenhahn \& Weigert (1994). ${ }^{(c)}$ Pizzolato et al. (2003). ${ }^{(d)}$ Matt et al. (2012). ${ }^{(e)}$ Matt et al. (2015).

${ }^{(f)}$ See et al. (2017).

\section{Appendix F: Observational trends used in this work and their caveats}

Table F.1. Observational trends used in this work and their caveats.

\begin{tabular}{lll}
\hline \hline Ingredient & References & Caveats \\
\hline Skumanich law & $\begin{array}{l}\text { Skumanich (1972) } \\
\text { Gallet \& Bouvier (2015) }\end{array}$ & $\begin{array}{l}\text { Uncertainty in the core-envelope coupling timescale dependency. Possible } \\
\text { break of gyrochronology for evolved stars. }\end{array}$ \\
& van Saders et al. (2016) & \\
\hline Wind braking torque & Matt et al. (2015) & $\begin{array}{l}\text { Slows rotators with lower stellar masses spinning too fast compared to the } \\
\text { one observed in the Kepler field. Possible dependency on the metallicity. }\end{array}$ \\
\hline$L_{X}-$ Ro relationship & $\begin{array}{l}\text { Pizzolato et al. (2003) } \\
\text { Wright et al. (2011) } \\
\text { Reiners et al. (2014) }\end{array}$ & Uncertainties in the trend obtained due to observational biases. \\
\hline ZDI studies & $\begin{array}{l}\text { Reiners (2012) } \\
\text { Vidotto et al. (2014) }\end{array}$ & $\begin{array}{l}\text { Only large-scale unsigned magnetic flux can be measured, missing small- } \\
\text { scale field, observational uncertainties. }\end{array}$ \\
& See et al. (2017) & $\begin{array}{l}\text { Assumed interstellar medium parameters, limited number of systems, } \\
\text { large scatter in scaling law. }\end{array}$ \\
\hline Astrospheric wind measurements & Wood et al. (2002, 2005) & \\
\hline
\end{tabular}

